\title{
An extragradient-like approximation method for variational inequalities and fixed point problems
}

\author{
Lu-Chuan Ceng ${ }^{1,2}$, Qamrul Hasan Ansari ${ }^{3}$, Ngai-Ching Wong ${ }^{4^{*}}$ and Jen-Chih Yao ${ }^{4,5}$
}

\author{
* Correspondence: wong@math. \\ nsysu.edu.tw \\ ${ }^{4}$ Department of Applied \\ Mathematics, National Sun Yat-Sen \\ University, Kaohsiung 80424, \\ Taiwan \\ Full list of author information is \\ available at the end of the article
}

\begin{abstract}
The purpose of this paper is to investigate the problem of finding a common element of the set of fixed points of an asymptotically strict pseudocontractive mapping in the intermediate sense and the set of solutions of a variational inequality problem for a monotone and Lipschitz continuous mapping. We introduce an extragradient-like iterative algorithm that is based on the extragradient-like approximation method and the modified Mann iteration process. We establish a strong convergence theorem for two sequences generated by this extragradient-like iterative algorithm. Utilizing this theorem, we also design an iterative process for finding a common fixed point of two mappings, one of which is an asymptotically strict pseudocontractive mapping in the intermediate sense and the other taken from the more general class of Lipschitz pseudocontractive mappings.

1991 MSC: 47H09; 47J20.

Keywords: extragradient-like approximation method, modified Mann iteration process, variational inequality, asymptotically strict pseudocontractive mapping in the intermediate sense, fixed point, monotone mapping, strong convergence, demiclosedness principle
\end{abstract}

\section{Introduction}

Let $H$ be a real Hilbert space whose inner product and norm are denoted by $\langle\cdot, \cdot\rangle$ and $\|$ - $\|$, respectively, and let $C$ be a nonempty closed convex subset of $H$. Corresponding to an operator $A: C \rightarrow H$ and set $C$, the variational inequality problem $\operatorname{VIP}(A, C)$ is defined as follows:

Find $\bar{x} \in C$ such that $\langle A \bar{x}, y-\bar{x}\rangle \geq 0, \quad \forall y \in C$.

The set of solutions of $\operatorname{VIP}(A, C)$ is denoted by $\Omega$. It is well known that if $A$ is a strongly monotone and Lipschitz-continuous mapping on $C$, then the $\operatorname{VIP}(A, C)$ has a unique solution. Not only the existence and uniqueness of a solution are important topics in the study of the $\operatorname{VIP}(A, C)$ but also how to compute a solution of the $\operatorname{VIP}(A$, $C)$ is important. For applications and further details on $\operatorname{VIP}(A, C)$, we refer to [1-4] and the references therein.

The set of fixed points of a mapping $S$ is denoted by $\operatorname{Fix}(S)$, that is, $\operatorname{Fix}(S)=\{x \in H$ : $S x=x\}$.

For finding an element of $F(S) \cap \Omega$ under the assumption that a set $C \subset H$ is nonempty, closed and convex, a mapping $S: C \rightarrow C$ is nonexpansive and a mapping $A: C$

(C) 2011 Ceng et al; licensee Springer. This is an Open Access article distributed under the terms of the Creative Commons Attribution License (http://creativecommons.org/licenses/by/2.0), which permits unrestricted use, distribution, and reproduction in any medium, provided the original work is properly cited. 
$\rightarrow H$ is $\beta$-inverse-strongly monotone, Takahashi and Toyoda [5] proposed an iterative scheme and proved that the sequence generated by the proposed scheme converges weakly to a point $z \in F(S) \cap \Omega$ if $F(S) \cap \Omega \neq \varnothing$.

Recently, motivated by the idea of Korpelevich's extragradient method [6], Nadezhkina and Takahashi [7] introduced an iterative scheme, called extragradient method, for finding an element of $F(S) \cap \Omega$ and established the weak convergence result. Very recently, inspired by the work in [7], Zeng and Yao [8] introduced an iterative scheme for finding an element of $F(S) \cap \Omega$ and obtained the weak convergence result. The viscosity approximation method for finding a fixed point of a given nonexpansive mapping was proposed by Moudafi [9]. He proved the strong convergence of the sequence generated by the proposed method to a unique solution of some variational inequality. $\mathrm{Xu}$ [10] extended the results of [9] to the more general version. Later on, Ceng and Yao [11] also introduced an extragradient-like approximation method, which is based on the above extragradient method and viscosity approximation method, and proved the strong convergence result under certain conditions.

An iterative method for the approximation of fixed points of asymptotically nonexpansive mappings was developed by Schu [12]. Iterative methods for the approximation of fixed points of asymptotically nonexpansive mappings have been further studied in $[13,14]$ and the references therein. The class of asymptotically nonexpansive mappings in the intermediate sense was introduced by Bruck et al. [15]. The iterative methods for the approximation of fixed points of such types of non-Lipschitzian mappings have been further studied in [16-18]. On the other hand, Kim and Xu [19] introduced the concept of asymptotically $\kappa$-strict pseudocontractive mappings in a Hilbert space and studied the weak and strong convergence theorems for this class of mappings. Sahu et al. [20] considered the concept of asymptotically $\kappa$-strict pseudocontractive mappings in the intermediate sense, which are not necessarily Lipschitzian. They proposed modified Mann iteration process and proved its weak convergence for an asymptotically $\kappa$-strict pseudocontractive mapping in the intermediate sense.

Very recently, Ceng et al. [21] established the strong convergence of viscosity approximation method for a modified Mann iteration process for asymptotically strict pseudocontractive mappings in intermediate sense and then proved the strong convergence of general CQ algorithm for asymptotically strict pseudocontractive mappings in intermediate sense. They extended the concept of asymptotically strict pseudocontractive mappings in intermediate sense to Banach space setting, called nearly asymptotically $\kappa$-strict pseudocontractive mapping in intermediate sense.

They also established the weak convergence theorems for a fixed point of a nearly asymptotically $\kappa$-strict pseudocontractive mapping in intermediate sense which is not necessarily Lipschitzian.

In this paper, we propose and study an extragradient-like iterative algorithm that is based on the extragradient-like approximation method in [11] and the modified Mann iteration process in [20]. We apply the extragradient-like iterative algorithm to designing an iterative scheme for finding a common fixed point of two nonlinear mappings. Here, we remind the reader of the following facts: (i) the modified Mann iteration process in [[20], Theorem 3.4] is extended to develop the extragradient-like iterative algorithm for finding an element of $F(S) \cap \Omega$; (ii) the extragradient-like iterative algorithm 
is very different from the extragradient-like iterative scheme in [11] since the class of mappings $S$ in our scheme is more general than the class of nonexpansive mappings.

\section{Preliminaries}

Throughout the paper, unless otherwise specified, we assume that $H$ is a real Hilbert space whose inner product and norm are denoted by $\langle\cdot, \cdot\rangle$ and $\|\cdot\|$, respectively, and $C$ is a nonempty closed convex subset of $H$. The set of fixed points of a mapping $S$ is denoted by $\operatorname{Fix}(S)$, that is, $\operatorname{Fix}(S)=\{x \in H: S x=x\}$. We write $x_{n} \rightarrow x$ to indicate that the sequence $\left\{x_{n}\right\}$ converges weakly to $x$. The sequence $\left\{x_{n}\right\}$ converges strongly to $x$ is denoted by $x_{n} \rightarrow x$.

Recall that a mapping $S: C \rightarrow C$ is said to be $L$-Lipschitzian if there exists a constant $L \geq 0$ such that $\|S x-S y\| \leq L\|x-y\|, \forall x, y \in C$. In particular, if $L \in[0,1)$, then $S$ is called a contraction on $C$; if $L=1$, then $S$ is called a nonexpansive mapping on $C$. The mapping $S: C \rightarrow C$ is called pseudocontractive if

$$
\|S x-S y\|^{2} \leq\|x-y\|^{2}+\|(I-S) x-(I-S) y\|^{2}, \quad \forall x, y \in C .
$$

A mapping $A: C \rightarrow H$ is called

(i) monotone if

$$
\langle A x-A y, x-y\rangle \geq 0, \quad \forall x, y \in C ;
$$

(ii) $\beta$-inverse-strongly monotone [22,23] if there exists a positive constant $\beta$ such that

$$
\langle A x-A y, x-y\rangle \geq \beta\|A x-A y\|^{2}, \quad \forall x, y \in C .
$$

It is obvious that if $A$ is $\beta$-inverse-strongly monotone, then $A$ is monotone and Lipschitz continuous.

It is easy to see that if a mapping $S: C \rightarrow C$ is nonexpansive, then the mapping $A=$ $I-S$ is $1 / 2$-inverse-strongly monotone; moreover, $F(S)=\Omega$ (see, e.g., [5]). At the same time, if a mapping $S: C \rightarrow C$ is pseudocontractive and $L$-Lipschitz continuous, then the mapping $A=(I-S)$ is monotone and $L+1$-Lipschitz continuous; moreover, $F(S)=$ $\Omega$ (see, e.g., [[24], proof of Theorem 4.5]).

Definition 2.1. Let $C$ be a nonempty subset of a normed space $X$. A mapping $S: C$ $\rightarrow C$ is said to be

(a) asymptotically nonexpansive [25] if there exists a sequence $\left\{k_{n}\right\}$ of positive numbers such that $\lim _{n \rightarrow \infty} K_{n}=1$ and

$$
\left\|S^{n} x-S^{n} y\right\| \leq k_{n}\|x-y\|, \quad \forall n \geq 1, \forall x, y \in C ;
$$

(b) asymptotically nonexpansive in the intermediate sense [15] provided $S$ is uniformly continuous and

$$
\limsup _{n \rightarrow \infty} \sup _{x, y \in C}\left(\left\|S^{n} x-S^{n} y\right\|-\|x-y\|\right) \leq 0 ;
$$

(c) uniformly Lipschitzian if there exists a constant $L>0$ such that

$$
\left\|S^{n} x-S^{n} y\right\| \leq L\|x-y\|, \quad \forall n \geq 1, \forall x, y \in C .
$$


It is clear that every nonexpansive mapping is asymptotically nonexpansive and every asymptotically nonexpansive mapping is uniformly Lipschitzian.

The class of asymptotically nonexpansive mappings was introduced by Goebel and Kirk [25] as an important generalization of the class of nonexpansive mappings. The existence of fixed points of asymptotically nonexpansive mappings was proved by Goebel and Kirk [25] as below:

Theorem 2.1. [[25], Theorem 1] If $C$ is a nonempty closed convex bounded subset of a uniformly convex Banach space, then every asymptotically nonexpansive mapping $S$ : $C \rightarrow C$ has a fixed point in $C$.

Definition 2.2. [19] A mapping $S: C \rightarrow C$ is said to be an asymptotically $\kappa$-strict pseudocontractive mapping with sequence $\left\{\gamma_{n}\right\}$ if there exist a constant $\kappa \in[0,1)$ and a sequence $\left\{\gamma_{n}\right\}$ in $[0, \infty)$ with $\lim _{n \rightarrow \infty} \gamma_{n}=0$ such that

$$
\left\|S^{n} x-S^{n} y\right\|^{2} \leq\left(1+\gamma_{n}\right)\|x-y\|^{2}+\kappa\left\|x-S^{n} x-\left(y-S^{n} y\right)\right\|^{2}, \quad \forall n \geq 1, \forall x, y \in C .
$$

It is important to note that every asymptotically $\kappa$-strict pseudocontractive mapping with sequence $\left\{\gamma_{n}\right\}$ is a uniformly L-Lipschitzian mapping with $L=\sup \left\{\frac{\kappa+\sqrt{1+(1-\kappa) \gamma_{n}}}{1+\kappa}: n \geq 1\right\}$.

Definition 2.3. [20] A mapping $S: C \rightarrow C$ is said to be an asymptotically $\kappa$-strict pseudocontractive mapping in the intermediate sense with sequence $\left\{\gamma_{n}\right\}$ if there exist a constant $\kappa \in[0,1)$ and a sequence $\left\{\gamma_{n}\right\}$ in $[0, \infty)$ with $\lim _{n \rightarrow \infty} \gamma_{n}=0$ such that

$$
\limsup _{n \rightarrow \infty} \sup _{x, y \in C}\left(\left\|S^{n} x-S^{n} y\right\|^{2}-\left(1+\gamma_{n}\right)\|x-y\|^{2}-\kappa\left\|x-S^{n} x-\left(y-S^{n} y\right)\right\|^{2}\right) \leq 0 .
$$

Put

$$
c_{n}:=\max \left\{0, \sup _{x, y \in C}\left(\left\|S^{n} x-S^{n} y\right\|^{2}-\left(1+\gamma_{n}\right)\|x-y\|^{2}-\kappa\left\|x-S^{n} x-\left(y-S^{n} y\right)\right\|^{2}\right)\right\} .
$$

Then, $c_{n} \geq 0(\forall n \geq 1), c_{n} \rightarrow 0(n \rightarrow \infty)$ and (2.2) reduces to the relation

$$
\left\|S^{n} x-S^{n} y\right\|^{2} \leq\left(1+\gamma_{n}\right)\|x-y\|^{2}+\kappa\left\|x-S^{n} x-\left(y-S^{n} y\right)\right\|^{2}+c_{n}, \quad \forall n \geq 1, \forall x, y \in C .
$$

Whenever $c_{n}=0$ for all $n \geq 1$ in (2.3), then $S$ is an asymptotically $\kappa$-strict pseudocontractive mapping with sequence $\left\{\gamma_{n}\right\}$.

For every point $x \in H$, there exists a unique nearest point in $C$, denoted by $P_{C} x$, such that

$$
\left\|x-P_{C} x\right\| \leq\|x-y\|, \quad \forall y \in C .
$$

$P_{C}$ is called the metric projection of $H$ onto $C$. Recall that the inequality holds

$$
\left\langle x-P_{C} x, P_{C} x-y\right\rangle \geq 0, \quad \forall x \in H, y \in C .
$$

Moreover, it is equivalent to

$$
\left\|P_{C} x-P_{C} y\right\|^{2} \leq\left\langle P_{C} x-P_{C} y, x-y\right\rangle, \quad \forall x, y \in H ;
$$

it is also equivalent to

$$
\|x-y\|^{2} \geq\left\|x-P_{C} x\right\|^{2}+\left\|y-P_{C} x\right\|^{2}, \quad \forall x \in H, y \in C .
$$


It is easy to see that $P_{C}$ is a nonexpansive mapping from $H$ onto $C$; see, e.g., [26] for further detail.

Lemma 2.1. Let $A: C \rightarrow H$ be a monotone mapping. Then,

$$
u \in \Omega \quad \Leftrightarrow \quad u=P_{C}(u-\lambda A u), \quad \forall \lambda>0 .
$$

Lemma 2.2. Let $H$ be a real Hilbert space. Then, the following hold:

$$
\|x-y\|^{2}=\left.|| x\left\|^{2}-\right\| y\right|^{2}-2\langle x-y, y\rangle, \quad \forall x, y \in H .
$$

Lemma 2.3. [[20], Lemma 2.6] Let $S: C \rightarrow C$ be an asymptotically $\kappa$-strict pseudocontractive mapping in the intermediate sense with sequence $\left\{\gamma_{n}\right\}$. Then,

$$
\left\|S^{n} x-S^{n} y\right\| \leq \frac{1}{1-\kappa}\left(\kappa|| x-y \|+\sqrt{\left(1+(1-\kappa) \gamma_{n}\right)\|x-y\|^{2}+(1-\kappa) c_{n}}\right)
$$

for all $x, y \in C$ and $n \geq 1$.

Lemma 2.4. [[20], Lemma 2.7] Let $S: C \rightarrow C$ be a uniformly continuous asymptotically $\kappa$-strict pseudocontractive mapping in the intermediate sense with sequence $\left\{\gamma_{n}\right\}$. Let $\left\{x_{n}\right\}$ be a sequence in $C$ such that $\left\|x_{n}-x_{n+1}\right\| \rightarrow 0$ and $\left\|x_{n}-S^{n} x_{n}\right\| \rightarrow 0$ as $n \rightarrow$ $\infty$. Then, $\left\|x_{n}-S x_{n}\right\| \rightarrow 0$ as $n \rightarrow \infty$.

Proposition 2.1 (Demiclosedness Principle). [[20], Proposition 3.1] Let $S: C \rightarrow C$ be a continuous asymptotically $\kappa$-strict pseudocontractive mapping in the intermediate sense with sequence $\left\{\gamma_{n}\right\}$. Then, $I-S$ is demiclosed at zero in the sense that if $\left\{x_{n}\right\}$ is a sequence in $C$ such that $x_{n} \rightarrow x \in C$ and $\lim \sup _{m \rightarrow \infty} \lim \sup _{n \rightarrow \infty}\left\|x_{n} S^{m} x_{n}\right\|=0$, then $(I-S) x=0$.

Proposition 2.2. [[20], Proposition 3.2] Let $S: C \rightarrow C$ be a continuous asymptotically $\kappa$-strict pseudocontractive mapping in the intermediate sense with sequence $\left\{\gamma_{n}\right\}$ such that $F(S) \neq \varnothing$. Then, $F(S)$ is closed and convex.

Remark 2.1. Propositions 2.1 and 2.2 give some basic properties of an asymptotically $\kappa$-strict pseudocontractive mapping in the intermediate sense with sequence $\left\{\gamma_{n}\right\}$. Moreover, Proposition 2.1 extends the demiclosedness principles studied for certain classes of nonlinear mappings in [19,27-29].

Lemma 2.5. [30]Let $(X,\langle\cdot\rangle$,$) be an inner product space. Then, for all x, y, z \in X$ and all $\alpha, \beta, \gamma \in[0,1]$ with $\alpha+\beta+\gamma=1$, we have

$$
\|\alpha x+\beta y+\gamma z\|^{2}=\alpha\|x\|^{2}+\beta\|y\|^{2}+\gamma\|z\|^{2}-\alpha \beta\|x-\gamma\|^{2}-\alpha \gamma\|x-z\|^{2}-\beta \gamma\|y-z\|^{2} .
$$

Lemma 2.6. [[31], Lemma 2.5] Let $\left\{s_{n}\right\}$ be a sequence of nonnegative real numbers satisfying

$$
s_{n+1} \leq\left(1-\bar{\alpha}_{n}\right) s_{n}+\bar{\alpha}_{n} \bar{\beta}_{n}+\bar{\gamma}_{n}, \quad \forall n \geq 1,
$$

where $\left\{\bar{\alpha}_{n}\right\},\left\{\bar{\beta}_{n}\right\}$, and $\left\{\bar{\gamma}_{n}\right\}$ satisfy the conditions:

(i) $\left\{\bar{\alpha}_{n}\right\} \subset[0,1], \sum_{n=1}^{\infty} \bar{\alpha}_{n}=\infty$, or equivalently, $\prod_{n=1}^{\infty}\left(1-\bar{\alpha}_{n}\right)=0$;

(ii) $\lim \sup _{n \rightarrow \infty} \bar{\beta}_{n} \leq 0$;

(iii) $\bar{\gamma}_{n} \geq 0(n \geq 1), \sum_{n=1}^{\infty} \bar{\gamma}_{n}<\infty$.

Then, $\lim _{n \rightarrow \infty} s_{n}=0$. 
Lemma 2.7. [32] Let $\left\{x_{n}\right\}$ and $\left\{z_{n}\right\}$ be bounded sequences in a Banach space $X$ and let $\left\{\mathrm{Q}_{n}\right\}$ be a sequence in $[0,1]$ with $0<\lim _{\inf _{n \rightarrow \infty}} \mathrm{\varrho}_{n} \leq \lim _{\sup _{n \rightarrow \infty}} \mathrm{\varrho}_{n} \leq 1$. Suppose that

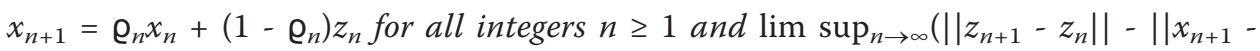
$\left.x_{n}||\right) \leq 0$. Then, $\lim _{n \rightarrow \infty}|| z_{n}-x_{n}||=0$.

The following lemma can be easily proved, and therefore, we omit the proof.

Lemma 2.8. In a real Hilbert space $H$, there holds the inequality

$$
\|x+y\|^{2} \leq\|x\|^{2}+2\langle y, x+y\rangle, \quad \forall x, y \in H .
$$

A set-valued mapping $T: H \rightarrow 2^{H}$ is called monotone if for all $x, y \in H, f \in T x$ and $g \in T y$ imply $\langle x-y, f-g\rangle \geq 0$. A monotone mapping $T: H \rightarrow 2^{H}$ is maximal if its graph $G(T)$ is not properly contained in the graph of any other monotone mapping. It is known that a monotone mapping $T$ is maximal if and only if for $(x, f) \in H \times H,\langle x$ $y, f-g\rangle \geq 0$ for all $(y, g) \in G(T)$ implies $f \in T x$. Let $A: C \rightarrow H$ be a monotone, $L$ Lipschitz continuous mapping and let $N_{C} v$ be the normal cone to $C$ at $v \in C$, i.e., $N_{C} v$ $=\{w \in H:\langle v-u, w\rangle \geq 0, \forall u \in C\}$. Define

$$
T v= \begin{cases}A v+N_{C} v & \text { if } v \in C, \\ \varnothing & \text { if } v \notin C .\end{cases}
$$

It is known that in this case $T$ is maximal monotone, and $0 \in T v$ if and only if $v \in$ $\Omega$; see [33].

\section{Extragradient-like approximation method and strong convergence results}

Let $A: C \rightarrow H$ be a monotone and $L$-Lipschitz continuous mapping, $f: C \rightarrow C$ be a contraction with contractive constant $\alpha \in(0,1)$ and $S: C \rightarrow C$ be an asymptotically $\kappa$-strict pseudocontractive mapping in the intermediate sense with sequence $\left\{\gamma_{n}\right\}$. In this paper, we introduce an extragradient-like iterative algorithm that is based on the extragradient-like approximation method in [11] and the modified Mann iteration process in [20]:

$$
\left\{\begin{array}{l}
x_{1}=x \in C \text { chosen arbitrary, } \\
y_{n}=\left(1-\mu_{n}\right) x_{n}+\mu_{n} P_{C}\left(x_{n}-\lambda_{n} A x_{n}\right), \\
t_{n}=P_{C}\left(x_{n}-\lambda_{n} A y_{n}\right), \\
x_{n+1}=\left(1-\alpha_{n}-\beta_{n}-v_{n}\right) x_{n}+\alpha_{n} f\left(y_{n}\right)+\beta_{n} t_{n}+v_{n} S^{n} t_{n}, \quad \forall n \geq 1,
\end{array}\right.
$$

where $\left\{\lambda_{n}\right\}$ is a sequence in $(0,1)$ with $\sum_{n=1}^{\infty} \lambda_{n}<\infty$, and $\left\{\alpha_{n}\right\},\left\{\beta_{n}\right\},\left\{\mu_{n}\right\}$ and $\left\{v_{n}\right\}$ are sequences in $[0,1]$ satisfying the following conditions:

(A1) $\alpha_{n}+\beta_{n}+v_{n} \leq 1$ for all $n \geq 1$;

(A2) $\lim _{n \rightarrow \infty} \alpha_{n}=0, \sum_{n=1}^{\infty} \alpha_{n}=\infty$;

(A3) $\kappa<\lim \inf _{n \rightarrow \infty} \beta_{n} \leq \lim \sup _{n \rightarrow \infty} \beta_{n}<1$;

(A4) $\sum_{n=1}^{\infty} v_{n}=\infty$.

The following result shows the strong convergence of the sequences $\left\{x_{n}\right\},\left\{y_{n}\right\}$ generated by the scheme (3.1) to the same point $q=P_{F(S) \cap \Omega} f(q)$ if and only if $\left\{A x_{n}\right\}$ is bounded, $\left\|\left(I-S^{n}\right) x_{n}\right\| \rightarrow 0$ and $\lim _{\inf _{n \rightarrow \infty}}\left\langle A x_{n}, y-x_{n}\right\rangle \geq 0$ for all $y \in C$.

Theorem 3.1. Let $A: C \rightarrow H$ be a monotone and L-Lipschitz continuous mapping, $f$ : $C \rightarrow C$ be a contraction with contractive constant $\alpha \in(0,1)$ and $S: C \rightarrow C$ be a uniformly continuous asymptotically $\kappa$-strict pseudocontractive mapping in the 
intermediate sense with sequence $\left\{\gamma_{n}\right\}$ such that $F(S) \cap \Omega \neq \varnothing$ and $\sum_{n=1}^{\infty} \gamma_{n}<\infty$. Let $\left\{x_{n}\right\},\left\{y_{n}\right\}$ be the sequences generated by (3.1), where $\left\{\lambda_{n}\right\}$ is a sequence in $(0,1)$ with $\sum_{n=1}^{\infty} \lambda_{n}<\infty$, and $\left\{\alpha_{n}\right\},\left\{\beta_{n}\right\},\left\{\mu_{n}\right\}$ and $\left\{y_{n}\right\}$ are sequences in $[0,1]$ satisfying the conditions (A1)-(A4). Then, the sequences $\left\{x_{n}\right\},\left\{y_{n}\right\}$ converge strongly to the same point $q=$ $P_{F(S) \cap \Omega} f(q)$ if and only if $\left\{A x_{n}\right\}$ is bounded, $\left\|\left(I-S^{n}\right) x_{n}\right\| \rightarrow 0$ and $\lim _{\inf _{n \rightarrow \infty}}\left\langle A x_{n}, y-\right.$ $\left.x_{n}\right\rangle \geq 0$ for all $y \in C$.

Proof. "Necessity". Suppose that the sequences $\left\{x_{n}\right\},\left\{y_{n}\right\}$ converge strongly to the same point $q=P_{F(S) \cap \Omega} f(q)$. Then from the $L$-Lipschitz continuity of $A$, it follows that $\left\{A x_{n}\right\}$ is bounded, and for each $y \in C$ :

$$
\begin{aligned}
& \left|\left\langle A x_{n}, y-x_{n}\right\rangle-\langle A q, y-q\rangle\right| \\
& \quad \leq\left|\left\langle A x_{n}, y-x_{n}\right\rangle-\left\langle A x_{n}, y-q\right\rangle\right|+\left|\left\langle A x_{n}, y-q\right\rangle-\langle A q, y-q\rangle\right| \\
& \quad=\left|\left\langle A x_{n}, q-x_{n}\right\rangle\right|+\left|\left\langle A x_{n}-A q, y-q\right\rangle\right| \\
& \quad \leq\left\|A x_{n}\left|\left\||| q-x_{n}\right\|+\left\|A x_{n}-A q\right\|\||| y-q\|\right|\right. \\
& \quad \leq\left\|A x_{n}\left|\left\||| q-x_{n}\right\|+L\right|\left|x_{n}-q\right|\right\| \mid y-q \| \rightarrow 0,
\end{aligned}
$$

which implies that

$$
\lim _{n \rightarrow \infty}\left\langle A x_{n}, y-x_{n}\right\rangle=\langle A q, y-q\rangle \geq 0, \quad \forall y \in C
$$

due to $q \in \Omega$. Furthermore, utilizing Lemma 2.3, we have

$$
\left\|S^{n} x_{n}-q\right\| \leq \frac{1}{1-\kappa}\left(\kappa\left\|x_{n}-q\right\|+\sqrt{\left(1+(1-\kappa) \gamma_{n}\right)\left\|x_{n}-q\right\|^{2}+(1-\kappa) c_{n}}\right) \rightarrow 0
$$

due to $x_{n} \rightarrow q, \gamma_{n} \rightarrow 0$ and $c_{n} \rightarrow 0$. Consequently, we conclude that for each $y \in C$

$$
\left\|S^{n} x_{n}-x_{n}\right\| \leq\left\|S^{n} x_{n}-q\right\|+\left\|x_{n}-q\right\| \rightarrow 0 .
$$

That is, $\left\|\left(I-S^{n}\right) x_{n}\right\| \rightarrow 0$.

"Sufficiency". Suppose that $\left\{A x_{n}\right\}$ is bounded, $\left\|\left(I-S^{n}\right) x_{n}\right\| \rightarrow 0$ and $\lim \inf _{n \rightarrow \infty}\left\langle A x_{n}\right.$, $\left.y-x_{n}\right\rangle \geq 0$ for all $y \in C$. Note that $\lim _{\inf _{n \rightarrow \infty}} \beta_{n}>\kappa$. Hence, we may assume, without loss of generality, that $\beta_{n}>\kappa$ for all $n \geq 1$.

Next, we divide the proof of the sufficiency into several steps.

STEP 1. We claim that $\left\{x_{n}\right\}$ is bounded. Indeed, put $t_{n}=P_{C}\left(x_{n}-\lambda_{n} A y_{n}\right)$ for all $n \geq 1$. Let $x^{*} \in F(S) \cap \Omega$. Then, $x^{*}=P_{C}\left(x^{*}-\lambda_{n} A x^{*}\right)$. Putting $x=x_{n}-\lambda_{n} A y_{n}$ and $y=x^{*}$ in (2.5), we obtain

$$
\begin{aligned}
\left\|t_{n}-x^{*}\right\|^{2} \leq & \left\|x_{n}-\lambda_{n} A y_{n}-x^{*}\right\|^{2}-\left\|x_{n}-\lambda_{n} A y_{n}-t_{n}\right\|^{2} \\
= & \left\|x_{n}-x^{*}\right\|^{2}-2 \lambda_{n}\left\langle A y_{n}, x_{n}-x^{*}\right\rangle+\lambda_{n}^{2}\left\|A y_{n}\right\|^{2} \\
& -\left\|x_{n}-t_{n}\right\|^{2}+2 \lambda_{n}\left\langle A y_{n}, x_{n}-t_{n}\right\rangle-\lambda_{n}^{2}\left\|A y_{n}\right\|^{2} \\
= & \left\|x_{n}-x^{*}\right\|^{2}+2 \lambda_{n}\left\langle A y_{n}, x^{*}-t_{n}\right\rangle-\left\|x_{n}-t_{n}\right\|^{2} \\
= & \left\|x_{n}-x^{*}\right\|^{2}-\left\|x_{n}-t_{n}\right\|^{2}-2 \lambda_{n}\left\langle A y_{n}-A x^{*}, y_{n}-x^{*}\right\rangle \\
& -2 \lambda_{n}\left\langle A x^{*}, y_{n}-x^{*}\right\rangle+2 \lambda_{n}\left\langle A y_{n}, y_{n}-t_{n}\right\rangle .
\end{aligned}
$$

Since $A$ is monotone and $x^{*}$ is a solution of $\operatorname{VIP}(A, C)$, we have

$$
\left\langle A y_{n}-A x^{*}, y_{n}-x^{*}\right\rangle \geq 0 \text { and }\left\langle A x^{*}, y_{n}-x^{*}\right\rangle \geq 0 \text {. }
$$


It follows from (3.2) that

$$
\begin{aligned}
\left\|t_{n}-x^{*}\right\|^{2} \leq & \left\|x_{n}-x^{*}\right\|^{2}-\left\|x_{n}-t_{n}\right\|^{2}+2 \lambda_{n}\left\langle A y_{n}, y_{n}-t_{n}\right\rangle \\
= & \left\|x_{n}-x^{*}\right\|^{2}-\left\|\left(x_{n}-y_{n}\right)+\left(y_{n}-t_{n}\right)\right\|^{2}+2 \lambda_{n}\left\langle A y_{n}, y_{n}-t_{n}\right\rangle \\
= & \left\|x_{n}-x^{*}\right\|^{2}-\left\|x_{n}-y_{n}\right\|^{2}-2\left\langle x_{n}-y_{n}, y_{n}-t_{n}\right\rangle-\left\|y_{n}-t_{n}\right\|^{2} \\
& +2 \lambda_{n}\left\langle A y_{n}, y_{n}-t_{n}\right\rangle \\
= & \left\|x_{n}-x^{*}\right\|^{2}-\left\|x_{n}-y_{n}\right\|^{2}-\left\|y_{n}-t_{n}\right\|^{2}+2\left\langle x_{n}-\lambda_{n} A y_{n}-y_{n}, t_{n}-y_{n}\right\rangle .
\end{aligned}
$$

Note that $x_{n} \in C$ for all $n \geq 1$ and that $y_{n}=\left(1-\mu_{n}\right) x_{n}+\mu_{n} P_{C}\left(x_{n}-\lambda_{n} A x_{n}\right)$. Hence, we have

$$
\begin{aligned}
& 2\left\langle x_{n}-\lambda_{n} A y_{n}-y_{n}, t_{n}-y_{n}\right\rangle \\
& \quad \leq 2\left\|x_{n}-\lambda_{n} A y_{n}-y_{n}\right\|\left\|t_{n}-y_{n}\right\| \leq\left\|x_{n}-\lambda_{n} A y_{n}-y_{n}\right\|^{2}+\left\|t_{n}-y_{n}\right\|^{2} \\
& \quad=\left\|x_{n}-y_{n}\right\|^{2}-2 \lambda_{n}\left\langle A y_{n}, x_{n}-y_{n}\right\rangle+\lambda_{n}^{2}\left\|A y_{n}\right\|^{2}+\left\|t_{n}-y_{n}\right\|^{2} \\
& \quad=\left\|x_{n}-y_{n}\right\|^{2}+\left\|t_{n}-y_{n}\right\|^{2}+2 \lambda_{n} \mu_{n}\left\langle A y_{n}, P_{C}\left(x_{n}-\lambda_{n} A x_{n}\right)-P_{C} x_{n}\right\rangle+\lambda_{n}^{2}\left\|A y_{n}\right\|^{2} \\
& \quad \leq\left\|x_{n}-y_{n}\right\|^{2}+\left\|t_{n}-y_{n}\right\|^{2}+2 \lambda_{n} \mu_{n}\left\|A y_{n}\right\|\left\|P_{C}\left(x_{n}-\lambda_{n} A x_{n}\right)-P_{C} x_{n}\right\|+\lambda_{n}^{2}\left\|A y_{n}\right\|^{2} \\
& \quad \leq\left\|x_{n}-y_{n}\right\|^{2}+\left\|t_{n}-y_{n}\right\|^{2}+2 \lambda_{n}^{2} \mu_{n}\left\|A y_{n}\right\|\left\|A x_{n}\right\|+\lambda_{n}^{2}\left\|A y_{n}\right\|^{2} .
\end{aligned}
$$

Since $\left\{A x_{n}\right\}$ is bounded and $A$ is $L$-Lipschitz continuous, we have

$$
\left\|A y_{n}-A x_{n}\right\| \leq L\left\|y_{n}-x_{n}\right\|=L \mu_{n}\left\|P_{C}\left(x_{n}-\lambda_{n} A x_{n}\right)-P_{C} x_{n}\right\| \leq L\left\|A x_{n}\right\|,
$$

and hence $\left\|A y_{n}\right\| \leq(1+L)\left\|A x_{n}\right\|$, which implies that $\left\{A y_{n}\right\}$ is bounded. Hence, we may assume that there exists a constant $M \geq \sup \left\{|| A x_{n}\|+\| A y_{n}\|+\| A x^{*} \|: n \geq 1\right\}$. Then, it follows from (3.4) that

$$
\begin{aligned}
2\left\langle x_{n}-\lambda_{n} A y_{n}-y_{n}, t_{n}-y_{n}\right\rangle & \leq\left\|x_{n}-y_{n}\right\|^{2}+\left\|t_{n}-y_{n}\right\|^{2}+\lambda_{n}^{2}\left(\left\|A x_{n}\right\|+\left\|A y_{n}\right\|\right)^{2} \\
& \leq\left\|x_{n}-y_{n}\right\|^{2}+\left\|t_{n}-y_{n}\right\|^{2}+\lambda_{n}^{2} M^{2} .
\end{aligned}
$$

This together with (3.3) implies that

$$
\begin{aligned}
\left\|t_{n}-x^{*}\right\|^{2} & \leq\left\|x_{n}-x^{*}\right\|^{2}-\left\|x_{n}-y_{n}\right\|^{2}-\left\|y_{n}-t_{n}\right\|^{2}+2\left\langle x_{n}-\lambda_{n} A y_{n}-y_{n}, t_{n}-y_{n}\right\rangle \\
& \leq\left\|x_{n}-x^{*}\right\|^{2}-\left\|x_{n}-y_{n}\right\|^{2}-\left\|y_{n}-t_{n}\right\|^{2}+\left\|x_{n}-y_{n}\right\|^{2}+\left\|t_{n}-y_{n}\right\|^{2}+\lambda_{n}^{2} M^{2} \\
& =\left\|x_{n}-x^{*}\right\|^{2}+\lambda_{n}^{2} M^{2}
\end{aligned}
$$

Observe that

$$
\begin{aligned}
&\left\|f\left(y_{n}\right)-x^{*}\right\|^{2} \\
& \leq \\
& \leq\left(\left\|f\left(y_{n}\right)-f\left(x^{*}\right)\right\|+\left\|f\left(x^{*}\right)-x^{*}\right\|\right)^{2} \\
& \leq\left(\alpha\left\|y_{n}-x^{*}\right\|+\left\|f\left(x^{*}\right)-x^{*}\right\|\right)^{2} \\
&=\left(\alpha\left\|y_{n}-x^{*}\right\|+(1-\alpha) \frac{\left\|f\left(x^{*}\right)-x^{*}\right\|}{1-\alpha}\right)^{2} \\
& \leq \alpha\left\|y_{n}-x^{*}\right\|^{2}+\frac{\left\|f\left(x^{*}\right)-x^{*}\right\|^{2}}{1-\alpha} \\
&= \alpha \|\left(1-\mu_{n}\right)\left(x_{n}-x^{*}\right)+\mu_{n}\left(P_{C}\left(x_{n}-\lambda_{n} A x_{n}\right)-P_{C}\left(x^{*}-\lambda_{n} A x^{*}\right) \|^{2}+\frac{\left\|f\left(x^{*}\right)-x^{*}\right\|^{2}}{1-\alpha}\right. \\
& \leq \alpha\left[\left(1-\mu_{n}\right)\left\|x_{n}-x^{*}\right\|^{2}+\mu_{n}\left\|P_{C}\left(x_{n}-\lambda_{n} A x_{n}\right)-P_{C}\left(x^{*}-\lambda_{n} A x^{*}\right)\right\|^{2}\right]+\frac{\left\|f\left(x^{*}\right)-x^{*}\right\|^{2}}{1-\alpha} \\
& \leq \alpha\left[\left(1-\mu_{n}\right)\left\|x_{n}-x^{*}\right\|^{2}+\mu_{n}\left\|\left(x_{n}-x^{*}\right)-\lambda_{n}\left(A x_{n}-A x^{*}\right)\right\|^{2}\right]+\frac{\left\|f\left(x^{*}\right)-x^{*}\right\|^{2}}{1-\alpha} \\
&= \alpha\left[\left(1-\mu_{n}\right)\left\|x_{n}-x^{*}\right\|^{2}+\mu_{n}\left(\left\|x_{n}-x^{*}\right\|^{2}-2 \lambda_{n}\left\langle x_{n}-x^{*}, A x_{n}-A x^{*}\right\rangle\right.\right. \\
&\left.+\lambda_{n}^{2}\left\|A x_{n}-A x^{*}\right\|^{2}\right]+\frac{\left\|f\left(x^{*}\right)-x^{*}\right\|^{2}}{1-\alpha} \\
& \leq \alpha\left[\left(1-\mu_{n}\right)\left\|x_{n}-x^{*}\right\|^{2}+\mu_{n}\left(\left\|x_{n}-x^{*}\right\|^{2}+\lambda_{n}^{2}\left\|A x_{n}-A x^{*}\right\|^{2}\right]+\frac{\left\|f\left(x^{*}\right)-x^{*}\right\|^{2}}{1-\alpha}\right. \\
& \leq \alpha\left\|x_{n}-x^{*}\right\|^{2}+\lambda_{n}^{2} M^{2}+\frac{\left\|f\left(x^{*}\right)-x^{*}\right\|^{2}}{1-\alpha} .
\end{aligned}
$$


Putting $\tau_{n}=\alpha_{n}+\beta_{n}+v_{n}$ and utilizing Lemma 2.5, we obtain from (3.5) and (3.6)

$$
\begin{aligned}
& \left\|x_{n+1}-x^{*}\right\|^{2} \\
& =\left\|\left(1-\alpha_{n}-\beta_{n}-v_{n}\right)\left(x_{n}-x^{*}\right)+\alpha_{n}\left(f\left(y_{n}\right)-x^{*}\right)+\beta_{n}\left(t_{n}-x^{*}\right)+v_{n}\left(S^{n} t_{n}-x^{*}\right)\right\|^{2} \\
& \leq\left(1-\tau_{n}\right)\left\|x_{n}-x^{*}\right\|^{2}+\tau_{n}\left\|\frac{\alpha_{n}}{\tau_{n}}\left(f\left(y_{n}\right)-x^{*}\right)+\frac{\beta_{n}}{\tau_{n}}\left(t_{n}-x^{*}\right)+\frac{\nu_{n}}{\tau_{n}}\left(S^{n} t_{n}-x^{*}\right)\right\|^{2} \\
& \leq\left(1-\tau_{n}\right)\left\|x_{n}-x^{*}\right\|^{2}+\tau_{n}\left[\frac{\alpha_{n}}{\tau_{n}}\left\|f\left(y_{n}\right)-x^{*}\right\|^{2}+\frac{\beta_{n}}{\tau_{n}}\left\|t_{n}-x^{*}\right\|^{2}+\frac{v_{n}}{\tau_{n}}\left\|S^{n} t_{n}-x^{*}\right\|^{2}\right. \\
& \left.-\frac{\beta_{n} v_{n}}{\tau_{n}^{2}}\left\|t_{n}-S^{n} t_{n}\right\|^{2}\right] \\
& =\left(1-\tau_{n}\right)|| x_{n}-x^{*}\left\|^{2}+\alpha_{n}\right\| f\left(y_{n}\right)-x^{*}\left\|^{2}+\beta_{n}\right\| t_{n}-x^{*}\left\|^{2}+v_{n}\right\| S^{n} t_{n}-x^{*} \|^{2} \\
& -\frac{\beta_{n} v_{n}}{\tau_{n}}\left\|t_{n}-S^{n} t_{n}\right\|^{2} \\
& \leq\left(1-\tau_{n}\right)\left\|x_{n}-x^{*}\right\|^{2}+\alpha_{n}\left\|f\left(y_{n}\right)-x^{*}\right\|^{2}+\beta_{n}\left\|t_{n}-x^{*}\right\|^{2} \\
& +v_{n}\left[\left(1+\gamma_{n}\right)\left\|t_{n}-x^{*}\right\|^{2}+\kappa\left\|t_{n}-S^{n} t_{n}\right\|^{2}+c_{n}\right]-\frac{\beta_{n} v_{n}}{\tau_{n}}\left\|t_{n}-S^{n} t_{n}\right\|^{2} \\
& =\left(1-\tau_{n}\right)\left\|x_{n}-x^{*}\right\|^{2}+\alpha_{n}\left\|f\left(y_{n}\right)-x^{*}\right\|^{2}+\left(\beta_{n}+v_{n}+v_{n} \gamma_{n}\right)\left\|t_{n}-x^{*}\right\|^{2} \\
& +v_{n}\left(\kappa-\frac{\beta_{n}}{\tau_{n}}\right)\left\|t_{n}-S^{n} t_{n}\right\|^{2}+v_{n} c_{n} \\
& \leq\left(1-\tau_{n}\right)\left\|x_{n}-x^{*}\right\|^{2}+\alpha_{n}\left\|f\left(y_{n}\right)-x^{*}\right\|^{2}+\left(\beta_{n}+v_{n}+\gamma_{n}\right)\left\|t_{n}-x^{*}\right\|^{2}+v_{n} c_{n} \\
& \leq\left(1-\tau_{n}\right)\left\|x_{n}-x^{*}\right\|^{2}+\alpha_{n}\left[\alpha\left\|x_{n}-x^{*}\right\|^{2}+\lambda_{n}^{2} M^{2}+\frac{\left\|f\left(x^{*}\right)-x^{*}\right\|^{2}}{1-\alpha}\right] \\
& +\left(\beta_{n}+v_{n}+\gamma_{n}\right)\left(\left\|x_{n}-x^{*}\right\|^{2}+\lambda_{n}^{2} M^{2}\right)+v_{n} c_{n} \\
& =\left(1-(1-\alpha) \alpha_{n}+\gamma_{n}\right)\left\|x_{n}-x^{*}\right\|^{2}+\left(\alpha_{n}+\beta_{n}+\nu_{n}+\gamma_{n}\right) \lambda_{n}^{2} M^{2} \\
& +(1-\alpha) \alpha_{n} \frac{\left\|f\left(x^{*}\right)-x^{*}\right\|^{2}}{(1-\alpha)^{2}}+v_{n} c_{n} \\
& \leq\left(1-(1-\alpha) \alpha_{n}+\gamma_{n}\right) \max \left\{\left\|x_{n}-x^{*}\right\|^{2}, \frac{\left\|f\left(x^{*}\right)-x^{*}\right\|^{2}}{(1-\alpha)^{2}}\right\}+\left(1+\gamma_{n}\right) \lambda_{n}^{2} M^{2} \\
& +(1-\alpha) \alpha_{n} \max \left\{\left\|x_{n}-x^{*}\right\|^{2}, \frac{\left\|f\left(x^{*}\right)-x^{*}\right\|^{2}}{(1-\alpha)^{2}}\right\}+v_{n} c_{n} \\
& \leq\left(1+\gamma_{n}\right) \max \left\{\left\|x_{n}-x^{*}\right\|^{2}, \frac{\left\|f\left(x^{*}\right)-x^{*}\right\|^{2}}{(1-\alpha)^{2}}\right\}+2 M^{2} \lambda_{n}^{2}+v_{n} c_{n} .
\end{aligned}
$$

Now, let us show that for all $n \geq 1$

$$
\left\|x_{n+1}-x^{*}\right\|^{2} \leq\left(\prod_{j=1}^{n}\left(1+\gamma_{j}\right)\right)\left(\sum_{i=1}^{n}\left(2 M^{2} \lambda_{i}^{2}+v_{i} c_{i}\right)+\max \left\{\left\|x_{1}-x^{*}\right\|^{2}, \frac{\left\|f\left(x^{*}\right)-x^{*}\right\|^{2}}{(1-\alpha)^{2}}\right\}\right) .
$$

As a matter of fact, whenever $n=1$, from (3.7), we have

$$
\begin{aligned}
\left\|x_{2}-x^{*}\right\|^{2} & \leq\left(1+\gamma_{1}\right) \max \left\{\left\|x_{1}-x^{*}\right\|^{2}, \frac{\left\|f\left(x^{*}\right)-x^{*}\right\|^{2}}{(1-\alpha)^{2}}\right\}+2 M^{2} \lambda_{1}^{2}+v_{1} c_{1} \\
& \leq\left(1+\gamma_{1}\right)\left(\max \left\{\left\|x_{1}-x^{*}\right\|^{2}, \frac{\left\|f\left(x^{*}\right)-x^{*}\right\|^{2}}{(1-\alpha)^{2}}\right\}+2 M^{2} \lambda_{1}^{2}+v_{1} c_{1}\right) \\
& =\left(\prod_{j=1}^{1}\left(1+\gamma_{j}\right)\right)\left(\sum_{i=1}^{1}\left(2 M^{2} \lambda_{i}^{2}+v_{i} c_{i}\right)+\max \left\{\left\|x_{1}-x^{*}\right\|^{2}, \frac{\left\|f\left(x^{*}\right)-x^{*}\right\|^{2}}{(1-\alpha)^{2}}\right\}\right) .
\end{aligned}
$$

Assume that (3.8) holds for some $n \geq 1$. Consider the case of $n+1$. From (3.7), we obtain 


$$
\begin{aligned}
&\left\|x_{n+2}-x^{*}\right\|^{2} \\
& \leq\left(1+\gamma_{n+1}\right) \max \left\{\left\|x_{n+1}-x^{*}\right\|^{2}, \frac{\left\|f\left(x^{*}\right)-x^{*}\right\|^{2}}{(1-\alpha)^{2}}\right\}+2 M^{2} \lambda_{n+1}^{2}+v_{n+1} c_{n+1} \\
& \leq\left(1+\gamma_{n+1}\right)\left(\max \left\{\left\|x_{n+1}-x^{*}\right\|^{2}, \frac{\left\|f\left(x^{*}\right)-x^{*}\right\|^{2}}{(1-\alpha)^{2}}\right\}+2 M^{2} \lambda_{n+1}^{2}+v_{n+1} c_{n+1}\right) \\
& \leq\left(1+\gamma_{n+1}\right)\left(\operatorname { m a x } \left\{\left(\prod_{j=1}^{n}\left(1+\gamma_{j}\right)\right)\left(\sum_{i=1}^{n}\left(2 M^{2} \lambda_{i}^{2}+v_{i} c_{i}\right)+\max \left\{\left\|x_{1}-x^{*}\right\|^{2}, \frac{\left\|f\left(x^{*}\right)-x^{*}\right\|^{2}}{(1-\alpha)^{2}}\right\}\right)\right.\right. \\
& \frac{\left\|f\left(x^{*}\right)-x^{*}\right\|^{2}}{\left.\left.(1-\alpha)^{2}\right\}+2 M^{2} \lambda_{n+1}^{2}+v_{n+1} c_{n+1}\right)} \\
& \leq\left(1+\gamma_{n+1}\right)\left(\left(\prod_{j=1}^{n}\left(1+\gamma_{j}\right)\right)\left(\sum_{i=1}^{n}\left(2 M^{2} \lambda_{i}^{2}+v_{i} c_{i}\right)+\max \left\{\left\|x_{1}-x^{*}\right\|^{2}, \frac{\left\|f\left(x^{*}\right)-x^{*}\right\|^{2}}{(1-\alpha)^{2}}\right\}\right)\right. \\
&\left.+2 M^{2} \lambda_{n+1}^{2}+v_{n+1} c_{n+1}\right) \\
&=\left(\prod_{j=1}^{n+1}\left(1+\gamma_{j}\right)\right)\left(\sum_{i=1}^{n}\left(2 M^{2} \lambda_{i}^{2}+v_{i} c_{i}\right)+\max \left\{\left\|x_{1}-x^{*}\right\|^{2}, \frac{\left\|f\left(x^{*}\right)-x^{*}\right\|^{2}}{(1-\alpha)^{2}}\right\}\right) \\
&+\left(1+\gamma_{n+1}\right)\left(2 M^{2} \lambda_{n+1}^{2}+v_{n+1} c_{n+1}\right) \\
& \leq\left(\prod_{j=1}^{n+1}\left(1+\gamma_{j}\right)\right)\left(\sum_{i=1}^{n}\left(2 M^{2} \lambda_{i}^{2}+v_{i} c_{i}\right)+\max \left\{\left\|x_{1}-x^{*}\right\|^{2}, \frac{\left\|f\left(x^{*}\right)-x^{*}\right\|^{2}}{(1-\alpha)^{2}}\right\}\right) \\
&+\left(\prod_{j=1}^{n+1}\left(1+\gamma_{j}\right)\right)\left(2 M^{2} \lambda_{n+1}^{2}+v_{n+1} c_{n+1}\right) \\
&=\left(\prod_{j=1}^{n+1}\left(1+\gamma_{j}\right)\right)\left(\sum_{i=1}^{n+1}\left(2 M^{2} \lambda_{i}^{2}+v_{i} c_{i}\right)+\max \left\{\left\|x_{1}-x^{*}\right\|^{2}, \frac{\left\|f\left(x^{*}\right)-x^{*}\right\|^{2}}{(1-\alpha)^{2}}\right\}\right) . \\
&\left(1-x^{2}\right) \\
&
\end{aligned}
$$

This shows that (3.8) holds for the case of $n+1$. By induction, we know that (3.8) holds for all $n \geq 1$. Since $\sum_{n=1}^{\infty} \gamma_{n}<\infty, \sum_{n=1}^{\infty} \lambda_{n}^{2}<\infty$ and $\sum_{n=1}^{\infty} v_{n} c_{n}<\infty$, from (3.8) we deduce that for all $n \geq 1$

$$
\begin{aligned}
\left\|x_{n+1}-x^{*}\right\|^{2} & \leq\left(\prod_{j=1}^{n}\left(1+\gamma_{j}\right)\right)\left(\sum_{i=1}^{n}\left(2 M^{2} \lambda_{i}^{2}+v_{i} c_{i}\right)+\max \left\{\left\|x_{1}-x^{*}\right\|^{2}, \frac{\left\|f\left(x^{*}\right)-x^{*}\right\|^{2}}{(1-\alpha)^{2}}\right\}\right) \\
& \leq \exp \left(\sum_{j=1}^{n} \gamma_{j}\right)\left(\sum_{i=1}^{n}\left(2 M^{2} \lambda_{i}^{2}+v_{i} c_{i}\right)+\max \left\{\left\|x_{1}-x^{*}\right\|^{2}, \frac{\left\|f\left(x^{*}\right)-x^{*}\right\|^{2}}{(1-\alpha)^{2}}\right\}\right) \\
& \leq \exp \left(\sum_{j=1}^{\infty} \gamma_{j}\right)\left(\sum_{i=1}^{\infty}\left(2 M^{2} \lambda_{i}^{2}+v_{i} c_{i}\right)+\max \left\{\left\|x_{1}-x^{*}\right\|^{2}, \frac{\left\|f\left(x^{*}\right)-x^{*}\right\|^{2}}{(1-\alpha)^{2}}\right\}\right) .
\end{aligned}
$$

This implies that $\left\{x_{n}\right\}$ is bounded.

STEP 2. We claim that $\lim _{n \rightarrow \infty}|| x_{n+1}-x_{n}||=0$. Indeed, observe that

$$
\begin{aligned}
\left\|t_{n+1}-t_{n}\right\| & =\left\|P_{C}\left(x_{n+1}-\lambda_{n+1} A y_{n+1}\right)-P_{C}\left(x_{n}-\lambda_{n} A y_{n}\right)\right\| \\
& \leq\left\|\left(x_{n+1}-\lambda_{n+1} A y_{n+1}\right)-\left(x_{n}-\lambda_{n} A y_{n}\right)\right\| \\
& \leq\left\|x_{n+1}-x_{n}\right\|+\lambda_{n+1}\left\|A y_{n+1}\right\|+\lambda_{n}\left\|A y_{n}\right\| \\
& \leq\left\|x_{n+1}-x_{n}\right\|+\left(\lambda_{n}+\lambda_{n+1}\right) M
\end{aligned}
$$


and

$$
\begin{aligned}
\left\|y_{n+1}-y_{n}\right\|= & \|\left(1-\mu_{n+1}\right) x_{n+1}+\mu_{n+1} P_{C}\left(x_{n+1}-\lambda_{n+1} A x_{n+1}\right) \\
& -\left(1-\mu_{n}\right) x_{n}-\mu_{n} P_{C}\left(x_{n}-\lambda_{n} A x_{n}\right) \| \\
= & \|\left(1-\mu_{n+1}\right)\left(x_{n+1}-x_{n}\right)-\left(\mu_{n+1}-\mu_{n}\right) x_{n} \\
& +\mu_{n+1}\left(P_{C}\left(x_{n+1}-\lambda_{n+1} A x_{n+1}\right)-P_{C}\left(x_{n}-\lambda_{n} A x_{n}\right)\right) \\
& +\left(\mu_{n+1}-\mu_{n}\right) P_{C}\left(x_{n}-\lambda_{n} A x_{n}\right) \| \\
= & \|\left(1-\mu_{n+1}\right)\left(x_{n+1}-x_{n}\right)+\left(\mu_{n+1}-\mu_{n}\right)\left(P_{C}\left(x_{n}-\lambda_{n} A x_{n}\right)-x_{n}\right) \\
& +\mu_{n+1}\left(P_{C}\left(x_{n+1}-\lambda_{n+1} A x_{n+1}\right)-P_{C}\left(x_{n}-\lambda_{n} A x_{n}\right)\right) \| \\
\leq & \left(1-\mu_{n+1}\right)\left\|x_{n+1}-x_{n}\right\|+\left|\mu_{n+1}-\mu_{n}\right| \lambda_{n}\left\|A x_{n}\right\| \\
& +\mu_{n+1}\left[\left\|x_{n+1}-x_{n}\right\|+\lambda_{n+1}\left\|A x_{n+1}\right\|+\lambda_{n}\left\|A x_{n}\right\|\right] \\
\leq & \left\|x_{n+1}-x_{n}\right\|+\lambda_{n}\left\|A x_{n}\right\|+\lambda_{n+1}\left\|A x_{n+1}\right\|+\lambda_{n}\left\|A x_{n}\right\| \\
\leq & \left\|x_{n+1}-x_{n}\right\|+\left(2 \lambda_{n}+\lambda_{n+1}\right) M .
\end{aligned}
$$

Define a sequence $\left\{z_{n}\right\}$ by

$$
x_{n+1}=\varrho_{n} x_{n}+\left(1-\varrho_{n}\right) z_{n}, \quad \forall n \geq 1,
$$

where $\varrho_{n}=1-\alpha_{n}-\beta_{n}-v_{n}, \forall n \geq 1$. Then we have

$$
\begin{aligned}
z_{n+1}-z_{n}= & \frac{x_{n+2}-\varrho_{n+1} x_{n+1}}{1-\varrho_{n+1}}-\frac{x_{n+1}-\varrho_{n} x_{n}}{1-\varrho_{n}} \\
= & \frac{\alpha_{n+1} f\left(y_{n+1}\right)+\beta_{n+1} t_{n+1}+v_{n+1} S^{n+1} t_{n+1}}{1-\varrho_{n+1}}-\frac{\alpha_{n} f\left(y_{n}\right)+\beta_{n} t_{n}+v_{n} S^{n} t_{n}}{1-\varrho_{n}} \\
= & \frac{\alpha_{n+1}}{1-\varrho_{n+1}} f\left(y_{n+1}\right)-\frac{\alpha_{n}}{1-\varrho_{n}} f\left(y_{n}\right)+\frac{\beta_{n+1}}{1-\varrho_{n+1}}\left(t_{n+1}-t_{n}\right) \\
& +\left(\frac{\alpha_{n}+v_{n}}{1-\varrho_{n}}-\frac{\alpha_{n+1}+v_{n+1}}{1-\varrho_{n+1}}\right) t_{n}+\frac{v_{n+1}}{1-\varrho_{n+1}} S^{n+1} t_{n+1}-\frac{v_{n}}{1-\varrho_{n}} S^{n} t_{n} \\
= & \frac{\alpha_{n+1}}{1-\varrho_{n+1}}\left(f\left(y_{n+1}\right)-f\left(y_{n}\right)\right)+\left(\frac{\alpha_{n+1}}{1-\varrho_{n+1}}-\frac{\alpha_{n}}{1-\varrho_{n}}\right) f\left(y_{n}\right)+\frac{\beta_{n+1}}{1-\varrho_{n+1}}\left(t_{n+1}-t_{n}\right) \\
& +\left(\frac{\alpha_{n}+v_{n}}{1-\varrho_{n}}-\frac{\alpha_{n+1}+v_{n+1}}{1-\varrho_{n+1}}\right) t_{n}+\frac{v_{n+1}}{1-\varrho_{n+1}} S^{n+1} t_{n+1}-\frac{v_{n}}{1-\varrho_{n}} S^{n} t_{n} .
\end{aligned}
$$

From (3.9)-(3.11), we get

$$
\begin{aligned}
\left\|z_{n+1}-z_{n}\right\| \leq & \frac{\alpha_{n+1}}{1-\varrho_{n+1}}\left\|f\left(y_{n+1}\right)-f\left(y_{n}\right)\right\|+\mid \frac{\alpha_{n+1}}{1-\varrho_{n+1}}-\frac{\alpha_{n}}{1-\varrho_{n}}\left\|f\left(y_{n}\right)\right\|+\frac{\beta_{n+1}}{1-\varrho_{n+1}}\left\|t_{n+1}-t_{n}\right\| \\
& +\left|\frac{\alpha_{n}+v_{n}}{1-\varrho_{n}}-\frac{\alpha_{n+1}+v_{n+1}}{1-\varrho_{n+1}}\left\|\mid t_{n}\right\|+\frac{v_{n+1}}{1-\varrho_{n+1}}\left\|S^{n+1} t_{n+1}\right\|+\frac{v_{n}}{1-\varrho_{n}}\left\|S^{n} t_{n}\right\|\right. \\
\leq & \frac{\alpha \alpha_{n+1}}{1-\varrho_{n+1}}\left\|y_{n+1}-y_{n}\right\|+\left(\frac{\alpha_{n+1}+v_{n+1}}{1-\varrho_{n+1}}+\frac{\alpha_{n}+v_{n}}{1-\varrho_{n}}\right)\left(\left\|f\left(y_{n}\right)\right\|+\left\|t_{n}\right\|\right) \\
& +\frac{\beta_{n+1}}{1-\varrho_{n+1}}\left\|t_{n+1}-t_{n}\right\|+\frac{v_{n+1}}{1-\varrho_{n+1}}\left\|S^{n+1} t_{n+1}\right\|+\frac{v_{n}}{1-\varrho_{n}}\left\|S^{n} t_{n}\right\| \\
\leq & \frac{\alpha \alpha_{n+1}}{1-\varrho_{n+1}}\left[\left\|x_{n+1}-x_{n}\right\|+\left(2 \lambda_{n}+\lambda_{n+1}\right) M\right]+\left(\frac{\alpha_{n+1}+v_{n+1}}{1-\varrho_{n+1}}+\frac{\alpha_{n}+v_{n}}{1-\varrho_{n}}\right)\left(\left\|f\left(y_{n}\right)\right\|+\left\|t_{n}\right\|\right) \\
& +\frac{\beta_{n+1}}{1-\varrho_{n+1}}\left[\left\|x_{n+1}-x_{n}\right\|+\left(\lambda_{n}+\lambda_{n+1}\right) M\right]+\frac{v_{n+1}}{1-\varrho_{n+1}}\left\|S^{n+1} t_{n+1}\right\|+\frac{v_{n}}{1-\varrho_{n}}\left\|S^{n} t_{n}\right\| \\
\leq & \left\|x_{n+1}-x_{n}\right\|+\left(2 \lambda_{n}+\lambda_{n+1}\right) M+\left(\frac{\alpha_{n+1}+v_{n+1}}{1-\varrho_{n+1}}+\frac{\alpha_{n}+v_{n}}{1-\varrho_{n}}\right)\left(\left\|f\left(y_{n}\right)\right\|+\left\|t_{n}\right\|\right) \\
& +\frac{v_{n+1}}{1-\varrho_{n+1}}\left\|S^{n+1} t_{n+1}\right\|+\frac{v_{n}}{1-\varrho_{n}}\left\|S^{n} t_{n}\right\|
\end{aligned}
$$


which implies that

$$
\begin{aligned}
\left\|z_{n+1}-z_{n}\right\|-\left\|x_{n+1}-x_{n}\right\| \leq & \left(2 \lambda_{n}+\lambda_{n+1}\right) M+\left(\frac{\alpha_{n+1}+v_{n+1}}{1-\varrho_{n+1}}+\frac{\alpha_{n}+v_{n}}{1-\varrho_{n}}\right)\left(\left\|f\left(y_{n}\right)\right\|+\left\|t_{n}\right\|\right) \\
& +\frac{v_{n+1}}{1-\varrho_{n+1}}\left\|S^{n+1} t_{n+1}\right\|+\frac{v_{n}}{1-\varrho_{n}}\left\|S^{n} t_{n}\right\| .
\end{aligned}
$$

Note that the boundedness of $\left\{x_{n}\right\}$ implies that $\left\{f\left(x_{n}\right)\right\}$ is also bounded. Since

$$
\left\|y_{n}-x_{n}\right\|=\mu_{n}\left\|P_{C}\left(x_{n}-\lambda_{n} A x_{n}\right)-P_{C} x_{n}\right\| \leq \lambda_{n}\left\|A x_{n}\right\| \leq \lambda_{n} M \rightarrow 0,
$$

we know that $\left\{y_{n}\right\}$ is bounded and so is $\left\{f\left(y_{n}\right)\right\}$. Moreover, $\left\{t_{n}\right\}$ is bounded by (3.5). Now, utilizing Lemma 2.3, we obtain that

$$
\left\|S^{n} t_{n}-x^{*}\right\| \leq \frac{1}{1-\kappa}\left(\kappa\left\|t_{n}-x^{*}\right\|+\sqrt{\left(1+(1-\kappa) \gamma_{n}\right)\left\|t_{n}-x^{*}\right\|^{2}+(1-\kappa) c_{n}}\right) .
$$

Thus, from the boundedness of $\left\{t_{n}\right\}$, it follows that $\left\{S^{n} t_{n}\right\}$ is bounded. Also, note that conditions

(ii), (iii) imply

$$
\limsup _{n \rightarrow \infty} \frac{\alpha_{n}}{1-\varrho_{n}}=\limsup _{n \rightarrow \infty} \frac{\alpha_{n}}{\alpha_{n}+\beta_{n}+v_{n}} \leq \limsup _{n \rightarrow \infty} \frac{\alpha_{n}}{\beta_{n}}=0,
$$

and conditions (iii), (iv) lead to

$$
\limsup _{n \rightarrow \infty} \frac{v_{n}}{1-\varrho_{n}}=\limsup _{n \rightarrow \infty} \frac{v_{n}}{\alpha_{n}+\beta_{n}+v_{n}} \leq \limsup _{n \rightarrow \infty} \frac{v_{n}}{\beta_{n}}=0 .
$$

Thus, we deduce from (3.12) that

$$
\limsup _{n \rightarrow \infty}\left(\left\|z_{n+1}-z_{n}\right\|-\left\|x_{n+1}-x_{n}\right\|\right) \leq 0 .
$$

Since $\varrho_{n}=1-\alpha_{n}-\beta_{n}-v_{n}$, we know from conditions (ii), (iii), (iv) that

$$
0<\liminf _{n \rightarrow \infty} \varrho_{n} \leq \limsup _{n \rightarrow \infty} \varrho_{n}<1 .
$$

Thus, in terms of Lemma 2.7, we get $\lim _{n \rightarrow \infty}|| z_{n}-x_{n} \|=0$. Consequently,

$$
\lim _{n \rightarrow \infty}\left\|x_{n+1}-x_{n}\right\|=\lim _{n \rightarrow \infty}\left(1-\varrho_{n}\right)\left\|z_{n}-x_{n}\right\|=0 .
$$

STEP 3. We claim that $\lim _{n \rightarrow \infty}|| S x_{n}-x_{n}\left\|=\lim _{n \rightarrow \infty}|| S t_{n}-t_{n}\right\|=0$. Indeed, observe that

$$
\begin{aligned}
\left\|y_{n}-t_{n}\right\| & =\left\|\left(1-\mu_{n}\right)\left(P_{C} x_{n}-P_{C}\left(x_{n}-\lambda_{n} A y_{n}\right)\right)+\mu_{n}\left(P_{C}\left(x_{n}-\lambda_{n} A x_{n}\right)-P_{C}\left(x_{n}-\lambda_{n} A y_{n}\right)\right)\right\| \\
& \leq\left(1-\mu_{n}\right)\left\|P_{C} x_{n}-P_{C}\left(x_{n}-\lambda_{n} A y_{n}\right)\right\|+\mu_{n}\left\|P_{C}\left(x_{n}-\lambda_{n} A x_{n}\right)-P_{C}\left(x_{n}-\lambda_{n} A y_{n}\right)\right\| \\
& \leq \lambda_{n}\left\|A y_{n}\right\|+\lambda_{n}\left\|A x_{n}-A y_{n}\right\| \rightarrow 0
\end{aligned}
$$

and hence

$$
\left\|t_{n}-x_{n}\right\| \leq\left\|t_{n}-y_{n}\right\|+\left\|y_{n}-x_{n}\right\| \rightarrow 0 .
$$

Note that the following condition holds:

$$
\lim _{n \rightarrow \infty}\left\|S^{n} x_{n}-x_{n}\right\|=0 \text {. }
$$


Also, observe that

$$
\left\|S^{n} t_{n}-t_{n}\right\| \leq\left\|S^{n} t_{n}-S^{n} x_{n}\right\|+\left\|S^{n} x_{n}-x_{n}\right\|+\left\|x_{n}-t_{n}\right\| .
$$

Utilizing Lemma 2.3 and $t_{n}-x_{n} \rightarrow 0$, we have

$$
\left\|S^{n} t_{n}-S^{n} x_{n}\right\| \leq \frac{1}{1-\kappa}\left(\kappa|| t_{n}-x_{n} \|+\sqrt{\left(1+(1-\kappa) \gamma_{n}\right)\left\|t_{n}-x_{n}\right\|^{2}+(1-\kappa) c_{n}}\right) \rightarrow 0(3.17
$$

Thus from (3.15)-(3.17), we obtain

$$
\lim _{n \rightarrow \infty}\left\|S^{n} t_{n}-t_{n}\right\|=0 .
$$

In addition, from (3.9) and $x_{n+1}-x_{n} \rightarrow 0$, it follows that $t_{n+1}-t_{n \rightarrow 0}$. Therefore, utilizing the uniform continuity of $S$ and Lemma 2.4, we know that $\lim _{n \rightarrow \infty}\left\|S x_{n}-x_{n}\right\|=$ 0 and $\lim _{n \rightarrow \infty}|| S t_{n}-t_{n} \|=0$.

STEP 4. We claim that $\lim \sup _{n \rightarrow \infty}\left\langle f(q)-q, x_{n}-q\right\rangle \leq 0$. Indeed, we pick a subsequence $\left\{x_{n_{i}}\right\}$ of $\left\{x_{n}\right\}$ so that

$$
\limsup _{n \rightarrow \infty}\left\langle f(q)-q, x_{n}-q\right\rangle=\lim _{i \rightarrow \infty}\left\langle f(q)-q, x_{n_{i}}-q\right\rangle .
$$

Without loss of generality, let $x_{n_{i}} \rightarrow \hat{x} \in C$. Then, (3.19) reduces to

$$
\limsup _{n \rightarrow \infty}\left\langle f(q)-q, x_{n}-q\right\rangle=\langle f(q)-q, \hat{x}-q\rangle .
$$

In order to show $\langle f(q)-q, \hat{x}-q\rangle \leq 0$, it suffices to show that $\hat{x} \in F(S) \cap \Omega$. Since $S$ is uniformly continuous and $\left\|x_{n}-S x_{n}\right\| \rightarrow 0$, we see that $\left\|x_{n}-S^{m} x_{n}\right\| \rightarrow 0$ for all $m \geq$ 1. By Proposition 2.1, we obtain $\hat{x} \in F(S)$. Now let us show that $\hat{x} \in \Omega$. Let

$$
T v= \begin{cases}A v+N_{C} v & \text { if } v \in C, \\ \varnothing & \text { if } v \notin C .\end{cases}
$$

Then, $T$ is maximal monotone and $0 \in T v$ if and only if $v \in \Omega$; see [33]. Let $(v, w) \in$ $G(T)$. Then, we have $w \in T v=A v+N_{C} v$ and hence $w-A v \in N_{C} v$. Therefore, we have $\langle v-u, w-A v\rangle \geq 0$ for all $u \in C$. In particular, taking $u=x_{n_{i}}$, we get

$$
\begin{aligned}
\langle v-\hat{x}, w\rangle & =\liminf _{i \rightarrow \infty}\left\langle v-x_{n_{i}}, w\right\rangle \geq \liminf _{i \rightarrow \infty}\left\langle v-x_{n_{i}}, A v\right\rangle \\
& \left.=\liminf _{i \rightarrow \infty}\left[v-x_{n_{i}}, A v-A x_{n_{i}}\right\rangle+\left\langle v-x_{n_{i}}, A x_{n_{i}}\right\rangle\right] \\
& \geq \liminf _{i \rightarrow \infty}\left\langle v-x_{n_{i}}, A x_{n_{i}}\right\rangle \geq \liminf _{n \rightarrow \infty}\left\langle v-x_{n}, A x_{n}\right\rangle \geq 0
\end{aligned}
$$

and so $\langle v-\hat{x}, w\rangle \geq 0$. Since $T$ is maximal monotone, we have $\hat{x} \in T^{-1} 0$ and hence $\hat{x} \in \Omega$.

This shows that $\hat{x} \in F(S) \cap \Omega$. Therefore by the property of the metric projection, we derive $\langle f(q)-q, \hat{x}-q\rangle \leq 0$.

STEP 5. We claim that $\lim _{n \rightarrow \infty}\left\|x_{n}-q\right\|=0$ where $q=P_{F(S) \cap \Omega} f(q)$. Indeed, since $\left\{A x_{n}\right\},\left\{A y_{n}\right\},\left\{S^{n} t_{n}\right\}$ are bounded, we may assume that there exists a constant $M \geq \sup$ $\left\{|| A x_{n}\|+\| A y_{n}\|+\| A q\|+\| S^{n} t_{n}-q \|: n \geq 1 g\right.$. Then from (3.1), (3.5) and Lemma 2.8, we get 


$$
\begin{aligned}
&\left\|x_{n+1}-q\right\|^{2} \\
&=\left\|\left(1-\alpha_{n}-\beta_{n}-v_{n}\right)\left(x_{n}-q\right)+\alpha_{n}\left(f\left(y_{n}\right)-q\right)+\beta_{n}\left(t_{n}-q\right)+v_{n}\left(S^{n} t_{n}-q\right)\right\|^{2} \\
& \leq\left\|\left(1-\alpha_{n}-\beta_{n}-v_{n}\right)\left(x_{n}-q\right)+\beta_{n}\left(t_{n}-q\right)+v_{n}\left(S^{n} t_{n}-q\right)\right\|^{2}+2 \alpha_{n}\left\langle f\left(y_{n}\right)-q, x_{n+1}-q\right\rangle \\
& \leq {\left[\left(1-\alpha_{n}-\beta_{n}-v_{n}\right)\left\|x_{n}-q\right\|+\beta_{n}\left\|t_{n}-q\right\|+v_{n}\left\|S^{n} t_{n}-q\right\|\right]^{2}+2 \alpha_{n}\left\langle f\left(y_{n}\right)-q, x_{n+1}-q\right\rangle } \\
& \leq {\left[\left(1-\alpha_{n}-\beta_{n}-v_{n}\right)\left\|x_{n}-q\right\|+\beta_{n}\left(\left\|x_{n}-q\right\|+\lambda_{n} M\right)+v_{n} M\right]^{2}+2 \alpha_{n}\left\langle f\left(y_{n}\right)-q, x_{n+1}-q\right\rangle } \\
&= {\left[\left(1-\alpha_{n}-v_{n}\right)\left\|x_{n}-q\right\|+\left(\beta_{n} \lambda_{n}+v_{n}\right) M\right]^{2}+2 \alpha_{n}\left\langle f\left(y_{n}\right)-q, x_{n+1}-q\right\rangle } \\
& \leq {\left[\left(1-\alpha_{n}\right)\left\|x_{n}-q\right\|+\left(\lambda_{n}+v_{n}\right) M\right]^{2}+2 \alpha_{n}\left\langle f\left(y_{n}\right)-q, x_{n+1}-q\right\rangle } \\
&= {\left[\left(1-\alpha_{n}\right)\left\|x_{n}-q\right\|+\left(\lambda_{n}+v_{n}\right) M\right]^{2}+2 \alpha_{n}\left[\left\langle f\left(y_{n}\right)-f\left(x_{n}\right), x_{n+1}-q\right\rangle\right.} \\
&\left.+\left\langle f\left(x_{n}\right)-f(q), x_{n+1}-q\right\rangle+\left\langle f(q)-q, x_{n+1}-q\right\rangle\right] \\
& \leq\left(1-\alpha_{n}\right)^{2}\left\|x_{n}-q\right\|^{2}+\left(\lambda_{n}+v_{n}\right) M\left[2\left(1-\alpha_{n}\right)\left\|x_{n}-q\right\|+\left(\lambda_{n}+v_{n}\right) M\right] \\
&+2 \alpha_{n}\left[\alpha\left\|y_{n}-x_{n}\right\|\left\|x_{n+1}-q\right\|+\alpha\left\|x_{n}-q\right\|\left\|x_{n+1}-q\right\|+\left\langle f(q)-q, x_{n+1}-q\right\rangle\right] \\
& \leq\left(1-\alpha_{n}\right)^{2}\left\|x_{n}-q\right\|^{2}+\alpha_{n}\left[\left\|x_{n}-q\right\|^{2}+\left\|x_{n+1}-q\right\|^{2}\right]+2 \alpha_{n}\left[\alpha\left\|y_{n}-x_{n}\right\|\left\|x_{n+1}-q\right\| \|\right. \\
&\left.+\left\langle f(q)-q, x_{n+1}-q\right\rangle\right]+\left(\lambda_{n}+v_{n}\right) M\left[2\left\|x_{n}-q\right\|+\left(\lambda_{n}+v_{n}\right) M\right],
\end{aligned}
$$

which implies that

$$
\begin{aligned}
\left\|x_{n+1}-q\right\|^{2} \leq & \frac{\left(1-\alpha_{n}\right)^{2}+\alpha \alpha_{n}}{1-\alpha \alpha_{n}}\left\|x_{n}-q\right\|^{2}+\frac{2 \alpha_{n}}{1-\alpha \alpha_{n}}\left[\alpha\left\|y_{n}-x_{n}\right\|\left\|x_{n+1}-q\right\|+\left\langle f(q)-q, x_{n+1}-q\right\rangle\right] \\
& +\frac{1}{1-\alpha \alpha_{n}}\left(\lambda_{n}+v_{n}\right) M\left[2\left\|x_{n}-q\right\|+\left(\lambda_{n}+v_{n}\right) M\right] \\
\leq & \left(1-2(1-\alpha) \alpha_{n}+\frac{\alpha_{n}^{2}}{1-\alpha \alpha_{n}}\right)\left\|x_{n}-q\right\|^{2}+\frac{2 \alpha_{n}}{1-\alpha \alpha_{n}}\left[\alpha\left\|y_{n}-x_{n}\right\|\left\|x_{n+1}-q\right\|\right. \\
& \left.+\left\langle f(q)-q, x_{n+1}-q\right\rangle\right]+\frac{1}{1-\alpha \alpha_{n}}\left(\lambda_{n}+v_{n}\right) M\left[2\left\|x_{n}-q\right\|+\left(\lambda_{n}+v_{n}\right) M\right] \\
= & \left(1-2(1-\alpha) \alpha_{n}\right)\left\|x_{n}-q\right\|^{2}+2(1-\alpha) \alpha_{n} \\
& +\frac{1}{(1-\alpha)\left(1-\alpha \alpha_{n}\right)}\left[\frac{\alpha_{n}}{2}\left\|x_{n}-q\right\|^{2}+\alpha\left\|y_{n}-x_{n}\right\|\left\|x_{n+1}-q\right\|+\left\langle f(q)-q, x_{n+1}-q\right\rangle\right] \\
& +\frac{1}{1-\alpha \alpha_{n}}\left(\lambda_{n}+v_{n}\right) M\left[2\left\|x_{n}-q\right\|+\left(\lambda_{n}+v_{n}\right) M\right] .
\end{aligned}
$$

Note that $\lim _{n \rightarrow \infty} \alpha_{n}=0$ and $\sum_{n=1}^{\infty} 2(1-\alpha) \alpha_{n}=\infty$. Since $\lim \sup _{n \rightarrow \infty}\left\langle f(q)-q, x_{n+1}\right.$ $-q\rangle \leq 0, \lim _{n \rightarrow \infty}|| y_{n}-x_{n} \|=0$ and $\left\{x_{n}-q\right\}$ is bounded, we know that

$$
\limsup _{n \rightarrow \infty} \frac{1}{(1-\alpha)\left(1-\alpha \alpha_{n}\right)}\left[\frac{\alpha_{n}}{2}\left\|x_{n}-q\right\|^{2}+\alpha\left\|y_{n}-x_{n}\right\|\left\|x_{n+1}-q\right\|+\left\langle f(q)-q, x_{n+1}-q\right\rangle\right] \leq 0 \text {. }
$$

Also, since $\sum_{n=1}^{\infty} \lambda_{n}<\infty$ and $\sum_{n=1}^{\infty} v_{n}=\infty$, it is easy to see that

$$
\sum_{n=1}^{\infty} \frac{1}{1-\alpha_{n}}\left(\lambda_{n}+v_{n}\right) M\left[2\left\|x_{n}-q\right\|+\left(\lambda_{n}+v_{n}\right) M\right]<\infty .
$$

Therefore, according to Lemma 2.6, we deduce that from (3.20) that $\left\|x_{n}-q\right\| \rightarrow 0$. Further from $\left\|y_{n}-x_{n}\right\| \rightarrow 0$, we obtain $\left\|y_{n}-q\right\| \rightarrow 0$. This completes the proof.

In Theorem 3.1, if we put $v_{n}=0(\forall n \geq 1)$ and $S=I$ the identity mapping. Then, the iterative scheme (3.1) reduces to the following scheme:

$$
\left\{\begin{array}{l}
x_{1}=x \in C \text { chosen arbitrary, } \\
y_{n}=\left(1-\mu_{n}\right) x_{n}+\mu_{n} P_{C}\left(x_{n}-\lambda_{n} A x_{n}\right) \\
x_{n+1}=\left(1-\alpha_{n}-\beta_{n}\right) x_{n}+\alpha_{n} f\left(y_{n}\right)+\beta_{n} P_{C}\left(x_{n}-\lambda_{n} A y_{n}\right), \quad \forall n \geq 1
\end{array}\right.
$$

Moreover, it is easy to see that $\sum_{n=1}^{\infty} v_{n}=\infty$ and $\left\|\left(1-S^{n}\right) x_{n}\right\| \rightarrow 0$. Thus, we have following corollary.

Corollary 3.1. Let $A: C \rightarrow H$ be a monotone, L-Lipschitz continuous mapping, and $f$ $: C \rightarrow C$ be a contraction with contractive constant $\alpha L(0,1)$. Let $\Omega \neq \varnothing$. Let $\left\{x_{n}\right\},\left\{y_{n}\right\}$ 
be the sequences generated by (3.21), where $\left\{\lambda_{n}\right\}$ is a sequence in $(0,1)$ with $\sum_{n=1}^{\infty} \lambda_{n}<\infty$, and $\left\{\alpha_{n}\right\},\left\{\beta_{n}\right\}$ and $\left\{\mu_{n}\right\}$ are three sequences in $[0,1]$ satisfying the conditions:

(B1) $\alpha_{n}+\beta_{n} \leq 1$ for all $n \geq 1$,

(B2) $\lim _{n \rightarrow \infty} \alpha_{n}=0, \sum_{n=1}^{\infty} \alpha_{n}=\infty$;

(B3) $0<\lim \inf _{n \rightarrow \infty} \beta_{n} \leq \lim \sup _{n \rightarrow \infty} \beta_{n}<1$.

Then, the sequences $\left\{x_{n}\right\},\left\{y_{n}\right\}$ converge strongly to the same point $q=P_{\Omega} f(q)$ if and only if $\left\{A x_{n}\right\}$ is bounded and $\lim _{\inf _{n \rightarrow \infty}}\left\langle A x_{n}, y-x_{n}\right\rangle \geq 0$ for all $y \in C$.

If $A^{-1} 0=\Omega$ and $P_{H}=I$, the identity mapping of $H$, then the iterative scheme (3.1) reduces to the following iterative scheme:

$$
\left\{\begin{array}{l}
x_{1}=x \in H \text { chosen arbitrary, } \\
y_{n}=\left(1-\mu_{n}\right) x_{n}+\mu_{n}\left(x_{n}-\lambda_{n} A x_{n}\right), \\
t_{n}=x_{n}-\lambda_{n} A y_{n} \\
x_{n+1}=\left(1-\alpha_{n}-\beta_{n}-v_{n}\right) x_{n}+\alpha_{n} f\left(y_{n}\right)+\beta_{n} t_{n}+v_{n} S^{n} t_{n}, \quad \forall n \geq 1 .
\end{array}\right.
$$

The following corollary can be easily derived from Theorem 3.1.

Corollary 3.2. Let $f: H \rightarrow H$ be a contractive mapping with constant $\alpha \in(0,1), A$ : $H \rightarrow H$ be a monotone, L-Lipschitz continuous mapping and $S: H \rightarrow H$ be a uniformly continuous asymptotically $\kappa$-strict pseudocontractive mapping in the intermediate sense with sequence $\left\{\gamma_{n}\right\}$ such that $F(S) \cap A^{-1} 0 \neq \varnothing$ and $\sum_{n=1}^{\infty} \gamma_{n}<\infty$. Let $\left\{x_{n}\right\},\left\{y_{n}\right\}$ be the sequences generated by (3.22), where $\left\{\lambda_{n}\right\}$ is a sequence in $(0,1)$ with $\sum_{n=1}^{\infty} \lambda_{n}<\infty$, and $\left\{\alpha_{n}\right\},\left\{\beta_{n}\right\},\left\{\mu_{n}\right\}$ and $\left\{v_{n}\right\}$ are four sequences in $[0,1]$ satisfying the conditions (A1)(A4). Then, the sequences $\left\{x_{n}\right\},\left\{y_{n}\right\}$ converge strongly to the same point $q=P_{F(S) \cap A^{-1} 0} f(q)$ if and only if $\left\{A x_{n}\right\}$ is bounded, $\left\|\left(I-S^{n}\right) x_{n}\right\| \rightarrow 0$ and $\lim \inf _{n \rightarrow \infty}$ $\left\langle A x_{n}, y-x_{n}\right\rangle \geq 0$ for all $y \in H$.

Let $B: H \rightarrow 2^{H}$ be a maximal monotone mapping. Then, for any $x \in H$ and $r>0$, consider $J_{r}^{B} x=\{z \in H: z+r B z \ni x\}$. Such $J_{r}^{B} x$ is called the resolvent of $B$ and is denoted by $J_{r}^{B}=(I+r B)^{-1}$.

If we put $S=J_{r}^{B}$ and $P_{H}=I$, then the iterative scheme (3.1) reduces to the following scheme:

$$
\left\{\begin{array}{l}
x_{1}=x \in H \text { chosen arbitrary, } \\
y_{n}=\left(1-\mu_{n}\right) x_{n}+\mu_{n}\left(x_{n}-\lambda_{n} A x_{n}\right), \\
t_{n}=x_{n}-\lambda_{n} A y_{n} \\
x_{n+1}=\left(1-\alpha_{n}-\beta_{n}-v_{n}\right) x_{n}+\alpha_{n} f\left(y_{n}\right)+\beta_{n} t_{n}+v_{n}\left(J_{r}^{B}\right)^{n} t_{n}, \quad \forall n \geq 1 .
\end{array}\right.
$$

It is easy to see that $\kappa=0, \gamma_{n}=0$ and $c_{n}=0$ for all $n \geq 1$. Moreover, we have $A^{-1} 0=$ $\Omega$ and $F\left(J_{r}^{B}\right)=B^{-1} 0$. Thus, utilizing Theorem 3.1, we obtain the following corollary.

Corollary 3.3. Let $f: H \rightarrow H$ be a contractive mapping with constant $\alpha \in(0,1), A$ : $H \rightarrow H$ be a monotone, L-Lipschitz continuous mapping and $B: H \rightarrow 2^{H}$ be a maximal monotone mapping such that $A^{-1} 0 \cap B^{-1} \neq \varnothing$. Let $J_{r}^{B}$ be the resolvent of $B$ for each $r>0$. Let $\left\{x_{n}\right\},\left\{y_{n}\right\}$ be the sequences generated by (3.23), where $\left\{\lambda_{n}\right\}$ is a sequence in $(0,1)$ with $\sum_{n=1}^{\infty} \lambda_{n}<\infty$, and $\left\{\alpha_{n}\right\},\left\{\beta_{n}\right\},\left\{\mu_{n}\right\}$ and $\left\{v_{n}\right\}$ are four sequences in $[0,1]$ satisfying the conditions (A1)-(A4). Then, the sequences $\left\{x_{n}\right\},\left\{y_{n}\right\}$ converge strongly to the same 
point $q=P_{A^{-1} O \cap B^{-1} 0} f(q)$ if and only if $\left\{A x_{n}\right\}$ is bounded, $\left\|\left(I-\left(J_{r}^{B}\right)^{n}\right) x_{n}\right\| \rightarrow$ Oand lim $\inf _{n \rightarrow \infty}\left\langle A x_{n}, y-x_{n}\right\rangle \geq 0$ for all $y \in H$.

Corollary 3.4. Let $f: H \rightarrow H$ be a contractive mapping with constant $\alpha \in(0,1)$ and $A: H \rightarrow H$ be a monotone, L-Lipschitz continuous mapping such that $A^{-1} 0 \neq \varnothing$. Let $\left\{x_{n}\right\},\left\{y_{n}\right\}$ be the sequences generated by

$$
\left\{\begin{array}{l}
x_{1}=x \in H \text { chosen arbitrary, } \\
y_{n}=\left(1-\mu_{n}\right) x_{n}+\mu_{n}\left(x_{n}-\lambda_{n} A x_{n}\right), \\
x_{n+1}=\left(1-\alpha_{n}-\beta_{n}\right) x_{n}+\alpha_{n} f\left(y_{n}\right)+\beta_{n}\left(x_{n}-\lambda_{n} A y_{n}\right), \quad \forall n \geq 1,
\end{array}\right.
$$

where $\left\{\lambda_{n}\right\}$ is a sequence in $(0,1)$ with $\sum_{n=1}^{\infty} \lambda_{n}<\infty$, and $\left\{\alpha_{n}\right\},\left\{\beta_{n}\right\}$ and $\left\{\mu_{n}\right\}$ are three sequences in $[0,1]$ satisfying the conditions (B1)-(B3). Then, the sequences $\left\{x_{n}\right\},\left\{y_{n}\right\}$ converge strongly to the same point $q=P_{A^{-1}} f(q)$ if and only if $\left\{A x_{n}\right\}$ is bounded and lim $\inf _{n \rightarrow \infty}\left\langle A x_{n}, y-x_{n}\right\rangle \geq 0$ for all $y \in C$.

Proof. In Theorem 3.1, put $C=H, v_{n}=0(\forall n \geq 1)$ and $S=I$ the identity mapping of $H$. Then, we know that $\kappa=0, \gamma_{n}=0$ and $c_{n}=0$ for all $n \geq 1$. Moreover, we have $A^{-1} 0$ $=\Omega . P H=I$. In this case, it is easy to see that $\sum_{n=1}^{\infty} v_{n}=\infty$ and $\left\|\left(I-S^{n}\right) x_{n}\right\| \rightarrow 0$. Therefore, by Theorem 3.1, we obtain the desired conclusion. $\square$

We also know one more definition of a pseudocontractive mapping, which is equivalent to the definition given in the preliminaries. A mapping $S: C \rightarrow C$ is called pseudocontractive [26] if

$$
\langle S x-S y, x-y\rangle \leq\|x-y\|^{2}, \quad \forall x, y \in C .
$$

Obviously, the class of pseudocontractive mappings is more general than the class of nonexpansive mappings. For the class of pseudocontractive mappings, there are some nontrivial examples; see, e.g., [[24], p. 1239] for further details. In the following theorem, we introduce an iterative process that converges strongly to a common fixed point of two mappings, one of which is an asymptotically $\kappa$-strict pseudocontractive mapping in the intermediate sense with sequence $\left\{\gamma_{n}\right\}$ and the other Lipschitz continuous and pseudocontractive.

Theorem 3.2. Let $f: C \rightarrow C$ be a contractive mapping with constant $\alpha \in(0,1), T: C$ $\rightarrow C$ be a pseudocontractive, m-Lipschitz continuous mapping and $S: C \rightarrow C$ be a uniformly continuous asymptotically $\kappa$-strict pseudocontractive mapping in the intermediate sense with sequence $\left\{\gamma_{n}\right\}$ such that $F(S) \cap F(T) \neq \varnothing$ and $\sum_{n=1}^{\infty} \gamma_{n}<\infty$. Let $\left\{x_{n}\right\},\left\{y_{n}\right\}$ be the sequences generated by

$$
\left\{\begin{array}{l}
x_{1}=x \in C \text { chosen arbitrary, } \\
y_{n}=\left(1-\mu_{n}\right) x_{n}+\mu_{n} P_{C}\left(x_{n}-\lambda_{n} A x_{n}\right), \\
t_{n}=P_{C}\left(x_{n}-\lambda_{n} A y_{n}\right), \\
x_{n+1}=\left(1-\alpha_{n}-\beta_{n}-v_{n}\right) x_{n}+\alpha_{n} f\left(y_{n}\right)+\beta_{n} t_{n}+v_{n} S^{n} t_{n}, \quad \forall n \geq 1,
\end{array}\right.
$$

where $A=I-T,\left\{\lambda_{n}\right\}$ is a sequence in $(0,1)$ with $\sum_{n=1}^{\infty} \lambda_{n}<\infty$, and $\left\{\alpha_{n}\right\},\left\{\beta_{n}\right\},\left\{\mu_{n}\right\}$ and $\left\{v_{n}\right\}$ are four sequences in $[0,1]$ satisfying the conditions (A1)-(A4). Then, the sequences $\left\{x_{n}\right\},\left\{y_{n}\right\}$ converge strongly to the same point $q=P_{F(S) \cap F(T)} f(q)$ if and only if $\left\{A x_{n}\right\}$ is bounded, $\left\|\left(I-S^{n}\right) x_{n}\right\| \rightarrow 0$ and $\lim _{\inf _{n \rightarrow \infty}}\left\langle A x_{n}, y-x_{n}\right\rangle \geq 0$ for all $y \in C$.

Proof. Let $A=I-T$. Let us show that the mapping $A$ is monotone and $(m+1)$ Lipschitz continuous. Indeed, observe that 


$$
\langle A x-A y, x-y\rangle=\|x-y\|^{2}-\langle T x-T y, x-y\rangle \geq 0
$$

and

$$
\|A x-A y\|=\|x-y-(T x-T y)\| \leq\|x-y\|+\|T x-T y\| \leq(m+1)\|x-y\| .
$$

Now, let us show that $F(T)=\Omega$. Indeed, we have, for fixed $\lambda_{0} \in(0,1)$,

$$
T u=u \Leftrightarrow u=u-\lambda_{0} A u=P_{C}\left(u-\lambda_{0} A u\right) \Leftrightarrow\langle A u, y-u\rangle \geq 0, \quad \forall y \in C .
$$

By Theorem 3.1, we obtain the desired conclusion.

Theorem 3.3. Let $f: C \rightarrow C$ be a contractive mapping with constant $\alpha \in(0,1), T: C$ $\rightarrow C$ be a pseudocontractive, m-Lipschitz continuous mapping and $S: C \rightarrow C$ be a nonexpansive mapping such that $F(S) \cap F(T) \neq \varnothing$. Let $\left\{x_{n}\right\},\left\{y_{n}\right\}$ be the sequences generated by

$$
\left\{\begin{array}{l}
x_{1}=x \in C \text { chosen arbitrary, } \\
y_{n}=\left(1-\mu_{n}\right) x_{n}+\mu_{n} P_{C}\left(x_{n}-\lambda_{n} A x_{n}\right), \\
t_{n}=P_{C}\left(x_{n}-\lambda_{n} A y_{n}\right), \\
x_{n+1}=\left(1-\alpha_{n}-\beta_{n}-v_{n}\right) x_{n}+\alpha_{n} f\left(y_{n}\right)+\beta_{n} t_{n}+v_{n} S^{n} t_{n}, \quad \forall n \geq 1,
\end{array}\right.
$$

where $A=I-T,\left\{\lambda_{n}\right\}$ is a sequence in $(0,1)$ with $\sum_{n=1}^{\infty} \lambda_{n}<\infty$, and $\left\{\alpha_{n}\right\},\left\{\beta_{n}\right\},\left\{\mu_{n}\right\}$ and $\left\{v_{n}\right\}$

are sequences in $[0,1]$ satisfying the conditions (A1)-(A4). Then, the sequences $\left\{x_{n}\right\}$, $\left\{y_{n}\right\}$ converge strongly to the same point $q=P_{F(S) \cap F(T)} f(q)$ if and only if $\left\{A x_{n}\right\}$ is bounded, $\left\|\left(I-S^{n}\right) x_{n}\right\| \rightarrow 0$ and $\lim _{\inf _{n \rightarrow \infty}}\left\langle A x_{n}, y-x_{n}\right\rangle \geq 0$ for all $y \in C$.

Proof. Let $A=I-T$. In terms of the proof of Theorem 3.2, we know that $A$ is a monotone and $(m+1)$-Lipschitz continuous mapping such that $F(T)=\Omega$. Since $S$ is a nonexpansive mapping, we know that $\kappa=0, \gamma_{n}=0$ and $c_{n}=0$ for all $n \geq 1$. By Theorem 3.1, we obtain the desired conclusion.

\section{Acknowledgements}

In this research, the first author, L.-C. Ceng, was partially supported by the National Science Foundation of China (10771141), Ph.D. Program Foundation of Ministry of Education of China (20070270004), Science and Technology Commission of Shanghai Municipality Grant (075105118), and Shanghai Leading Academic Discipline Project (S30405). While, the third author, N.-C. Wong, and the last author, J.-C. Yao, were partially supported by the Taiwan NSC Grants 99-2115-M-110-007-MY3 and 99-2221-E-037-007-MY3, respectively.

\section{Author details}

${ }^{1}$ Department of Mathematics, Shanghai Normal University, Shanghai 200234, China ${ }^{2}$ Scientific Computing Key Laboratory Of Shanghai Universities, China 3 Department of Mathematics, Aligarh Muslim University, Aligarh 202002 , India ${ }^{4}$ Department of Applied Mathematics, National Sun Yat-Sen University, Kaohsiung 80424, Taiwan ${ }^{5}$ Center for General Education, Kaohsiung Medical University, Kaohsiung 80708, Taiwan

\section{Authors' contributions}

All authors contribute equally and significantly in writing this paper. All authors read and approved the final manuscript.

\section{Competing interests}

The authors declare that they have no competing interests.

Received: 2 February 2011 Accepted: 27 July 2011 Published: 27 July 2011

\section{References}

1. Baiocchi, C, Capelo, A: Variational and Quasivariational Inequalities, Application to Free Boundary Problems. Wiley, New York (1984)

2. Kinderlehrer, D, Stampacchia, G: An Introduction to Variational Inequalities and their Applications. Academic Press, New York (1980) 
3. Konnov, IV: Combined Relaxation Methods for Variational Inequalities, Lecture Notes in Mathematical Economics. Springer, Berlin (2000)

4. Patriksson, M: Nonlinear Programming and Variational Inequality Problems. Kluwer Academic Publishers, Dordrecht (1999)

5. Takahashi, W, Toyoda, M: Weak convergence theorems for nonexpansive mappings and monotone mappings. J Optim Theory Appl. 118, 417-428 (2003). doi:10.1023/A:1025407607560

6. Korpelevich, GM: The extragradient method for finding saddle points and other problems. Matecon. 12, 747-756 (1976)

7. Nadezhkina, N, Takahashi, W: Weak convergence theorem by an extragradient method for nonexpansive mappings and monotone mappings. J Optim Theory Appl. 128, 191-201 (2006). doi:10.1007/s10957-005-7564-z

8. Zeng, LC, Yao, JC: Strong convergence theorem by an extragradient method for fixed point problems and variational inequality problems. Taiwanese J Math. 10(5), 1293-1303 (2006)

9. Moudafi, A: Viscosity approximation methods for fixed-points problems. J Math Anal Appl. 241, 46-55 (2000). doi:10.1006/jmaa.1999.6615

10. Xu, HK: Viscosity approximation methods for nonexpansive mappings. J Math Anal Appl. 298, 279-291 (2004). doi:10.1016/j.jmaa.2004.04.059

11. Ceng, LC, Yao, JC: An extragradient-like approximation method for variational inequality problems and fixed point problems. Appl Math Comput. 190, 205-215 (2007). doi:10.1016/j.amc.2007.01.021

12. Schu, J: Iterative construction of fixed points of asymptotically nonexpansive mapping. J Math Anal Appl. 159, 407-413 (1991)

13. Ceng, LC, Xu, HK, Yao, JC: The viscosity approximation method for asymptotically nonexpansive mappings in Banach spaces. Nonlinear Anal. 69(4), 1402-1412 (2008). doi:10.1016/j.na.2007.06.040

14. Ceng, LC, Wong, NC, Yao, JC: Fixed point solutions of variational inequalities for a finite family of asymptotically nonexpansive mappings without common fixed point assumption. Comput Math Appl. 56, 2312-2322 (2008). doi:10.1016/j.camwa.2008.05.002

15. Bruck, RE, Kuczumow, T, Reich, S: Convergence of iterates of asymptotically nonexpansive mappings in Banach spaces with the uniform Opial property. Colloq Math. 65, 169-179 (1993)

16. Agarwal, RP, O'Regan, D, Sahu, DR: Iterative construction of fixed points of nearly asymptotically nonexpansive mappings. J Nonlinear Convex Anal. 8(1), 61-79 (2007)

17. Chidume, $\mathrm{CE}$, Shahzad, N, Zegeye, $\mathrm{H}$ : Convergence theorems for mappings which are asymptotically non-expansive in the intermediate sense. Numer Funct Anal Optim. 25, 239-257 (2004). doi:10.1081/NFA-120039611

18. Kim, GE, Kim, TH: Mann and Ishikawa iterations with errors for non-Lipschitzian mappings in Banach spaces. Comput Math Appl. 42, 1565-1570 (2001). doi:10.1016/S0898-1221(01)00262-0

19. Kim, TH, Xu, HK: Convergence of the modified Mann's iteration method for asymptotically strict pseudo-contractions. Nonlinear Anal. 68, 2828-2836 (2008). doi:10.1016/..na.2007.02.029

20. Sahu, DR, Xu, HK, Yao, JC: Asymptotically strict pseudocontractive mappings in the intermediate sense. Nonlinear Anal. 70, 3502-3511 (2009). doi:10.1016/j.na.2008.07.007

21. Ceng, LC, Ansari, QH, Yao, J-C: Strong and weak convergence theorems for asymptotically strict pseudo-contraction mappings in intermediate sense. J Nonlinear Convex Anal. 11(2), 283-308 (2010)

22. Browder, FE, Petryshyn, W: Construction of fixed points of nonlinear mappings in Hilbert space. J Math Anal Appl. 20, 747-756 (1967)

23. Liu, F, Nashed, MZ: Regularization of nonlinear ill-posed variational inequalities and convergence rates. Set-Valued Anal. 6, 313-344 (1998). doi:10.1023/A:1008643727926

24. Nadezhkina, N, Takahashi, W: Strong convergence theorem by a hybrid method for nonexpansive mappings and Lipschitz-continuous monotone mappings. SIAM J Optim. 16, 1230-1241 (2006). doi:10.1137/050624315

25. Goebel, K, Kirk, WA: A fixed point theorem for asymptotically nonexpansive mappings. Proc Am Math Soc. 35(1), 171-174 (1972). doi:10.1090/50002-9939-1972-0298500-3

26. Takahashi, W: Nonlinear Functional Analysis. Yokohama Publishers, Yokohama (2000)

27. Gornicki, J: Weak convergence theorems for asymptotically nonexpansive mappings in uniformly convex Banach spaces. Comment Math Univ Carolin. 30(2), 249-252 (1989)

28. Marino, $G, X u, H K$ : Weak and strong convergence theorems for strict pseudo-contractions in Hilbert spaces. J Math Anal Appl. 329, 336-346 (2007). doi:10.1016/j.jmaa.2006.06.055

29. $\mathrm{Xu}, \mathrm{HK}$ : Existence and convergence for fixed points for mappings of asymptotically nonexpansive type. Nonlinear Anal. 16, 1139-1146 (1991). doi:10.1016/0362-546X(91)90201-B

30. Osilike, MO, lgbokwe, DI: Weak and strong convergence theorems for fixed points of pseudocontractions and solutions of monotone type operator equations. Comput Math Appl. 40, 559-567 (2000). doi:10.1016/S0898-1221(00)00179-6

31. Xu, HK: Iterative algorithms for nonlinear operators. J London Math Soc. 66, 240-256 (2002). doi:10.1112/ S0024610702003332

32. Suzuki, T: Strong convergence of Krasnoselskii and Mann's type sequences for one-parameter nonexpansive semigroups without Bochner integrals. J Math Anal Appl. 305, 227-239 (2005). doi:10.1016/j.jmaa.2004.11.017

33. Rockafellar, RT: On the maximality of sums of nonlinear monotone operators. Trans Am Math Soc. 149, 75-88 (1970). doi:10.1090/S0002-9947-1970-0282272-5

doi:10.1186/1687-1812-2011-22

Cite this article as: Ceng et al: An extragradient-like approximation method for variational inequalities and fixed point problems. Fixed Point Theory and Applications 2011 2011:22. 